Review

\title{
Correction and suppression of reaching movements in the cerebral cortex: Physiological and neuropsychological aspects
}

\author{
Alexandra Battaglia-Mayer ${ }^{\mathrm{a}, 1}$, Tania Buiatti ${ }^{\mathrm{b}, 1}$, Roberto Caminiti ${ }^{\mathrm{a}, *, 1}$, \\ Stefano Ferraina ${ }^{\mathrm{a}, 1}$, Francesco Lacquaniti ${ }^{\mathrm{c}, \mathrm{d}, 1}$, Tim Shallice $^{\mathrm{b}, \mathrm{e}, 1}$ \\ a Department of Physiology and Pharmacology, SAPIENZA University of Rome, 00185 Rome, Italy \\ ${ }^{\mathrm{b}}$ Cognitive Neuropsychology and Neuroimaging Laboratory, International School for Advanced Studies (SISSA), 34136 Trieste, Italy \\ c Department of Neuromotor Physiology, Santa Lucia Foundation, 00179 Rome, Italy \\ ${ }^{\mathrm{d}}$ Department of Systems Medicine, University of Rome Tor Vergata, 00133 Rome, Italy \\ e Institute of Cognitive Neuroscience, University College London, London, UK
}

\section{A R T I C L E I N F O}

\section{Article history:}

Received 15 October 2013

Received in revised form 28 February 2014

Accepted 4 March 2014

\section{Keywords:}

Hand reaching

Movement correction

Movement suppression

Cerebral cortex control

Parieto-frontal system

\begin{abstract}
A B S T R A C T
Modification or suppression of reaches occurs in everyday life. We argue that a common modular architecture, based on similar neural structures and principles of kinematic and kinetic control, is used for both direct reaches and for their on-line corrections. When a reach is corrected, both the pattern of neural activity in parietal, premotor and motor cortex and the muscle synergies associated with the first movement can be smoothly blended or sharply substituted into those associated with the second one. Premotor cortex provides the early signaling for trajectory updating, while parietal and motor cortex provide the fine-grained encoding of hand kinematics necessary to reshape the motor plan. The cortical contribution to the inhibitory control of reaching is supported by the activity of a network of frontal areas. Premotor cortex has been proposed as a key structure for reaching suppression. Consistent with this, lesions in different nodes of this network result in different forms of motor deficits, such as Optic Ataxia in parietal patients, and commission errors in frontal ones.
\end{abstract}

(c) 2014 Elsevier Ltd. All rights reserved.

\section{Contents}

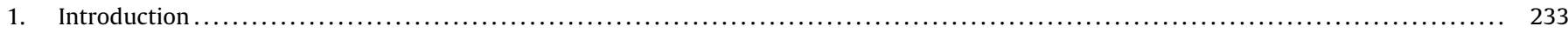

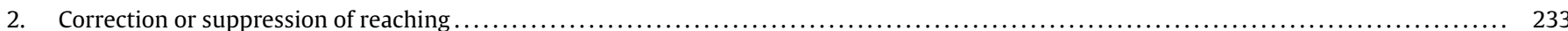

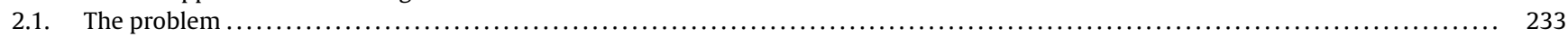

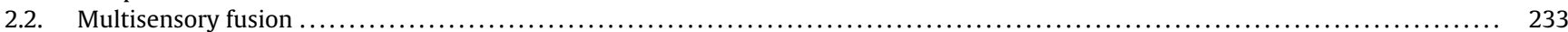

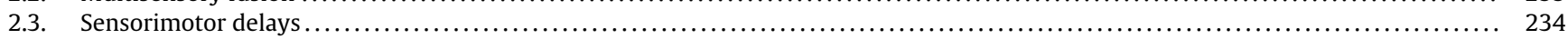

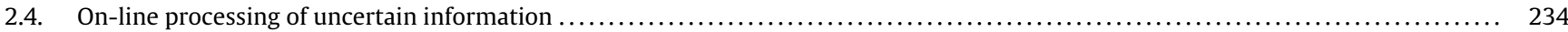

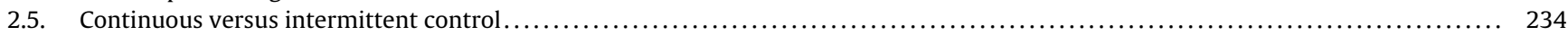

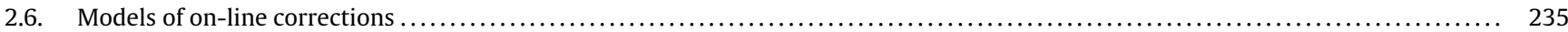

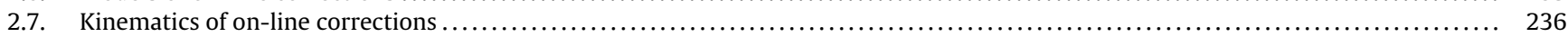

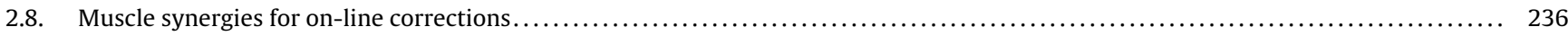

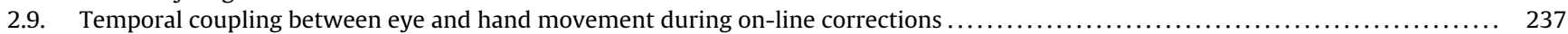

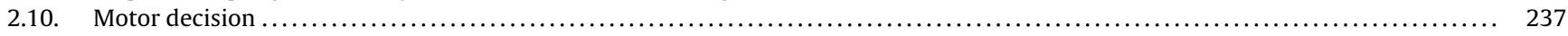

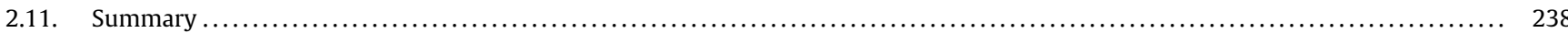

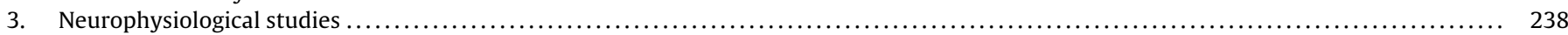

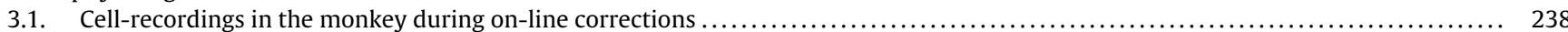

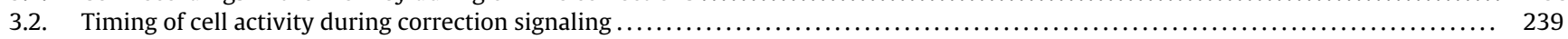

\footnotetext{
* Corresponding author at: Department of Physiology and Pharmacology, SAPIENZA University of Rome, Piazzale Aldo Moro 5, 00185 Rome, Italy. Tel.: +39 0649910967 ; fax: +3906 49910942 .

E-mail address: roberto.caminiti@uniroma1.it (R. Caminiti).

1 All authors contributed equally to this work.
} 
3.3. Potential neural mechanisms for movement correction...

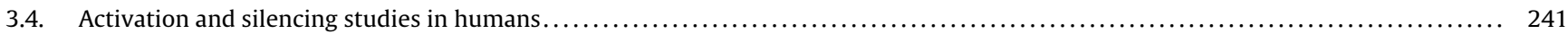

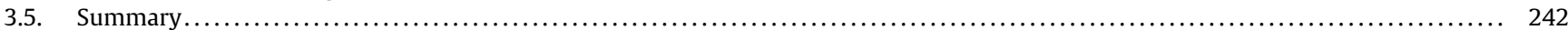

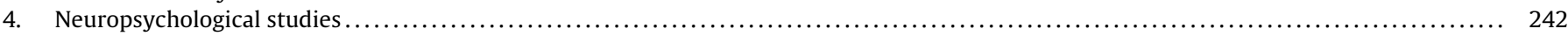

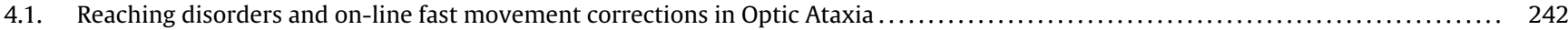

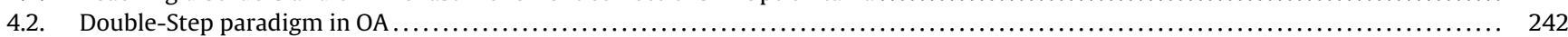

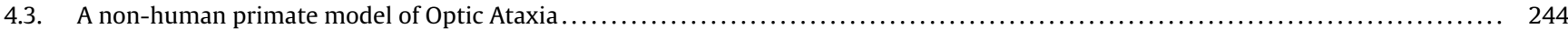

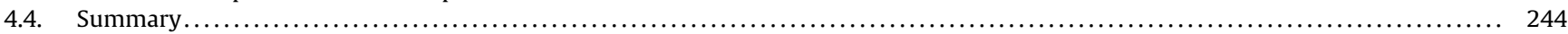

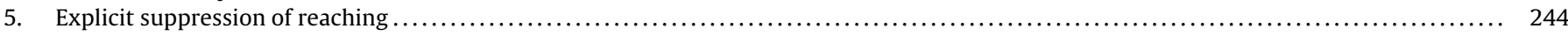

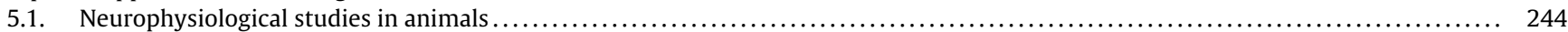

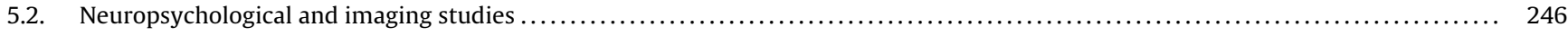

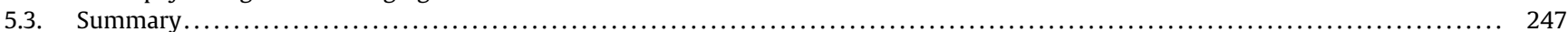

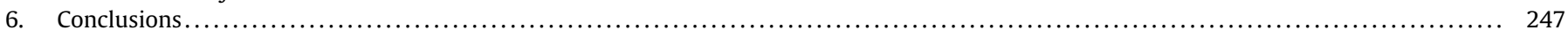

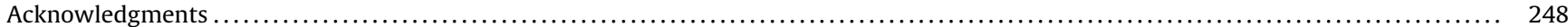

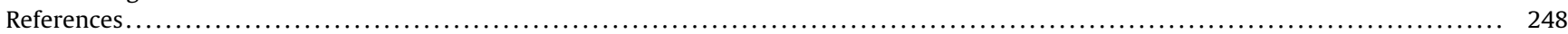

\section{Introduction}

Aside from locomotion, reaching provides the basic foundation for the great majority of the actions of humans and monkeys. Frequently, however, a reach must be modified in some way either just before or during execution, as the reached for object moves or there are signs that it might be inappropriate to touch. Therefore reaching must be a flexible form of motor behavior that requires planning and on-line control in order to modify or suppress the original motor plan or the ongoing hand movement, when needed. This flexibility can be studied at different levels of analysis, such as its behavioral characteristics, anatomical substrates, neurophysiological mechanisms and the consequences of brain lesions in patients. In this review, which is concerned with the cortical systems involved, we present experimental evidence for common, modular elements that are shared by both direct, unperturbed reaching and reaching modified because of either sudden target shifts or the need to stop the movement. Findings from both macaques and humans will be discussed, and functional parallels will be drawn based on the similarity in the anatomical structures involved in cortical motor control between these species. Different theoretical models and ideas will be illustrated and contrasted. We will argue that the overall set of finding is compatible with a relatively simple functional and anatomical model.

\section{Correction or suppression of reaching}

\subsection{The problem}

The problem of reaching a target, whether stationary or jumping, can be described in a straightforward manner. We define the motor error as the vector underlying desired hand movement, that is, the vector difference between target location and hand location. A successful reach involves nulling the motor error vector or reducing it to within a tolerance window defined by the task. Accordingly, signals about the target and limb state must be translated into neural commands appropriate to drive the arm toward the target. Conversely, stopping a reach to an unwanted target involves the translation of signals about the target into neural commands appropriate to suppress the arm movement. For all types of reaching behaviors, whether of generation, correction or suppression, there is the issue of how and when the corresponding decision is taken.

The deceiving simplicity of the problem formulation hides the formidable computational challenges faced by the brain for its solution (for reviews see Battaglia-Mayer et al., 2003; Crawford et al., 2011; Desmurget and Grafton, 2000; Franklin and Wolpert, 2011; Gold and Shadlen, 2007; Gomi, 2008; Lacquaniti, 1997; Sabes, 2011; Shadmehr and Mussa-Ivaldi, 2012; Shadmehr and Wise, 2005;
Soechting and Flanders, 1992). At the input side, state estimates for the target and limb require the fusion of multiple sensory signals encoded in disparate reference frames. Moreover, whenever the target or arm position changes, the corresponding signals must be updated, and similar updating is required for the other body parts, eyes, head or trunk, which contribute to encoding target and arm states. Sensory signals are typically noisy and delayed in time by hundreds of milliseconds relative to the monitored event, with both noise and delays varying considerably among sensory channels. At the output side, the central representations of the target and limb states must be transformed into specific patterns of activity of the arm muscles. Moreover, the implementation of motor commands is affected by transmission delays and noise. The motor implementation must also address the issue of redundancy, due to the fact that there are many more muscles (and motor units in each muscle) than degrees of freedom of movement and force at the hand. Despite the redundancy, the brain must find a unique solution of muscle activation (or deactivation) patterns for each task. Finally, input and output are not independent, because sensory feedback affects the output and, in turn, the output modifies the limb state and the corresponding sensory signals.

Here, we will review current ideas about how the brain deals with the challenges listed above. As a paradigmatic experimental approach to study reaching corrections, we will consider the Double-Step protocol, in which the target is displaced from an initial location to a new one at an unpredictable time. For movement suppression, we will consider the Go/No-Go task and the Stop (countermanding) protocols. In the former task, participants must reach a target in response to a Go signal, whereas they must stand still in response to randomly interspersed No-Go signals. In the countermanding task, instead, movement generation is directly pitted against movement suppression by requiring participants to cancel an impending response upon presentation of an infrequent Stop signal.

\subsection{Multisensory fusion}

In reaching, the integration of visual and proprioceptive signals generally allows most efficient localization of the stimuli and generation of the appropriate commands (Sabes, 2011). The initial stages involved in sensorimotor transformations deal with the issues of the different reference frames associated with different sensory channels, and with how multisensory information is merged into central representations of the reaching goal. It was previously thought that transforming different sensory signals into a common representation in a given reference frame should simplify reach planning. However, the search for a single common representation has not given consistent results, evidence having being provided in favor of each of the plausible reference frames 
(eye-, head-, body- or arm-centered). According to current views, instead, there is no need for a unique central representation: the presence of noisy sensory signals makes it advantageous to represent reach plans simultaneously in multiple reference frames (Battaglia-Mayer et al., 2003; McGuire and Sabes, 2009). McGuire and Sabes (2009) state that "when sensory signals are encoded in a statistically optimal manner, the same information is contained in multiple neural representations and there is no required relationship between the behavioral output and any single representation of the movement plan". Optimality in multisensory fusion could be based on a Bayesian combination of multi-cue information and prior state estimates, with a task-dependent reweighting of both sensory and prior information (Körding and Wolpert, 2004). Importantly, the weight placed on each sensory cue is proportional to the cue reliability: in other words, noisier cues are given smaller weights and this results in optimal state estimates.

\subsection{Sensorimotor delays}

The ability to make on-line corrections to reaching movements depends on the processing time of the changes in state. As we remarked before, neural communication at both sensory and motor sides is fraught by delays. In addition, there is a decision bottleneck. As a result of both transmission and decision delays, motor corrections may be retarded by an amount called the Psychological Refractory Period (PRP, Welford, 1952). When two step-stimuli are given in sequence, the PRP corresponds to the time interval over which the time to respond to the second step is prolonged relative to the time to respond to the first step (van de Kamp et al., 2013; Vince, 1948).

For the sake of simplicity, we focus on visuo-motor pathways, but similar issues exist for the other sensory-motor pathways. Transmission delays sum up as information is processed at the different stages of visuo-motor pathways, starting with the processing of optic information in the retina (Kane et al., 2011; Lamme and Roelfsema, 2000; Nijhawan, 2008). Visual information is transmitted from the retina to the lateral geniculate nucleus, and from there to area V1. For reaching to visual targets (Desmurget et al., 1999), information may be fed by V1 to the posterior parietal cortex (PPC) via the dorsal stream (Livingstone and Hubel, 1988; Maunsell et al., 1990; Merigan and Maunsell, 1993). In addition, however, achromatic signals can also reach PPC via the retinotectal pathway to the superior colliculus and pulvinar (Schiller and Malpeli, 1977; Schiller et al., 1979). Next, in monkeys cortico-cortical connections relay visual information from PPC to motor and premotor cortices, and there exist both feedforward and feedback connections in the parieto-frontal system (PFS, Averbeck et al., 2009; Caminiti et al., 1996). Overall, it may take between 85 and $150 \mathrm{~ms}$ for visual information to reach motor cortex (Lamme and Roelfsema, 2000). The conduction time from human motor cortex to arm and hand muscles is about $10-30 \mathrm{~ms}$, the time increasing with corticomuscular distance (Salenius et al., 1997). The electromechanical delay between EMG onset and mechanical force production in arm muscles is about $50 \mathrm{~ms}$. Finally, the inertia of the limb involved in reaching adds an additional subject-dependent delay. The net theoretical visuo-motor delay between a stimulus and the motor response - estimated by adding up these various sources of delay should be in the order of 150-300 ms for a typical arm movement.

However, in contrast with these theoretical estimates, behavioral studies show that corrections of on-going movements in response to an unpredictable change in visual target location can begin as early as $110 \mathrm{~ms}$ after the visual cue, but with a speed-accuracy trade-off (Brenner and Smeets, 1997; Day and Lyon, 2000; Prablanc and Martin, 1992). This latency is considerably less than that predicted by the estimates of neuromechanical delays summarized above. This discrepancy then raises the issue of how the CNS compensates for sensorimotor delays (Nijhawan, 2008; Schlag and Schlag-Rey, 2002). Neural compensation appears to take place already at the retinal level. Thus, in the isolated retinas of the salamander and rabbit, the population response of ganglion cells is extrapolated forward in time relative to the stimulus (Berry et al., 1999). There is also some evidence for neural compensation in central visual areas. Thus, the receptive field of a neural population in area V4 of the monkey has been shown to shift in the direction opposite to that of target motion, as if the cells had been recruited by a wave of activity preceding the target (Sundberg et al., 2006). In addition, delays can be compensated centrally by combining the sensory signals with the efference copies of the motor commands to the eyes and arm, as well as with internal models of the target and arm. Indeed, forward models are neural mechanisms which predict forthcoming sensory states (Wolpert and Miall, 1996). Because these models incorporate implicit knowledge of the kinematic and dynamic characteristics of the musculoskeletal system, they are able to predict the changes in position, velocity and force associated with a given motor command, provided the parameters of the controlled system (such as inertia, stiffness and viscosity) are correctly estimated and there are no perturbations. On the other hand, inverse internal models of the limb geometry and of the musculoskeletal dynamics can be used to map the desired hand trajectory into the muscle patterns driving the arm along that trajectory (Kawato, 1999). Both forward and inverse models can be used in internal error-correcting loops (Desmurget and Grafton, 2000; Kawato, 1999).

\subsection{On-line processing of uncertain information}

In daily life, as well as in many laboratory manipulations, target changes are probabilistic rather than deterministic. A probabilistic scenario is not easily accommodated within the classical view that, to correct a reaching movement in response to a target change, the brain uses only the information about the new target. An alternative view is that the new estimated target position is a weighted combination of the first and second targets. Izawa and Shadmehr (2008) tested this hypothesis by asking participants to reach toward a blurry target that occasionally jumped during the reach. Consistent with the probabilistic model, they found that the motor response to the second target was influenced by the uncertainty about the first target. Accordingly, they proposed that the brain makes predictions about the near future of sensory states and integrates the delayed sensory measures with the internal predictions to form an estimate of the current state.

Interestingly, in monkeys the discharge of PPC neurons faithfully reflects the amount of uncertainty of visual information, and the rate of increase in the neural discharge reflects the progressive accumulation of information about the target (Gold and Shadlen, 2007). After the discharge reaches a threshold, monkeys start the motor response. The hypothesis that the process of accumulating information must reach a threshold before initiation of action also accounts for the finding that human participants start their movements later when they are less certain of the exact location of the target (Izawa and Shadmehr, 2008).

\subsection{Continuous versus intermittent control}

One view about movement correction or suppression is that the motor command signals are updated continuously based on sensory feedback of the target and limb state (Day and Lyon, 2000; Desmurget et al., 1999; Goodale et al., 1986; Gritsenko et al., 2009; Pelisson et al., 1986; Prablanc and Martin, 1992; Saunders and Knill, 2003). According to this view, the motor error used to compute the motor commands is a time-varying variable, corresponding to 
the vector difference between instantaneous estimates of target location and hand location.

A different view is that, even though sensory feedback is continuous, the motor error and the ensuing commands are updated discontinuously or intermittently by the CNS (Beggs and Howarth, 1972; Loram et al., 2011; Navas and Stark, 1968; van de Kamp et al., 2013). In this case, correction and suppression would be discrete processes. It should be stressed at the outset that, despite the apparent limitations, intermittent control could be as effective as or more effective than continuous control, at least under some circumstances (Loram et al., 2011). This is because intermittency allows the integration (time-averaging) of incoming sensory information over extended time epochs, and may thus rely on less noisy estimates of the environment.

These two mechanisms may be difficult to disassociate behaviorally when the movement is smoothly adjusted at a short-latency from the change signal. The low-pass filtering properties of limb muscles and inertia tend to smooth out sharp transitions between discrete actions, giving the impression of a continuous response when in fact it is intermittent. However, robust evidence for intermittent control has been obtained in the case of tracking of unpredictable stimuli, a performance that deteriorates already beyond $1-2 \mathrm{~Hz}$, indicating a limited control bandwidth (Loram et al., 2009, 2011; Navas and Stark, 1968).

As far as reaching corrections are concerned, there is experimental evidence suggesting that the brain resorts to either continuous or intermittent control depending on the context. Two critical factors are the time interval intervening between the original and new target presentations, and the amplitude of target displacement in case of Double-Steps. Thus, if the target displacement is small and/or the time interval is short, an automatic correction mechanism can generate trajectory changes that are compatible with continuous on-line control (Desmurget et al., 1999; Goodale et al., 1986; Pelisson et al., 1986; Prablanc and Martin, 1992). Notice that many such cases correspond to target displacements occurring during the orienting saccade to the first target. Because of saccadic suppression, participants often remain unaware of the perturbation if its amplitude is small with respect to the amplitude of the saccade (Goodale et al., 1986). However, some doubts about the full automaticity of this process have recently been expressed, at least for tasks in which a voluntary modulation of the action is required. Indeed, it has been shown that fast in-flight corrections can be suppressed in the Double-Step reaching paradigm, if participants are told to ignore the new target location and continue reaching for the original one (Cameron et al., 2009; McIntosh et al., 2010; Striemer et al., 2010).

On the other hand, if the target displacement and/or the time interval are large, discrete rather than continuous correction mechanisms are triggered based on intermittent control. The speed required to reach the target is still another contextual factor that calls for the utilization of one or the other type of on-line control. Thus, slow to medium-speed movements are compatible with a continuous control. By contrast, ballistic reaching (i.e. very fast, impulsive movement) can hardly be sustained by continuous feedback, and instead progresses under either open-loop (feed-forward) control or intermittent feedback control. When intermittent control involves serial ballistic actions, these actions typically join smoothly with each other (Loram et al., 2011). In the case of reaching to shifting targets, intermittent updating of the commands results in the superposition of overlapping corrective sub-movements. Thus, when the target jumps away from its initial location, desired hand movements can be decomposed as the superposition of one trajectory from the start to the initial target and a subsequent trajectory from the initial to the final target. We will take up this issue when dealing with the mechanisms of control.
More specifically, in addition to the smooth modifications of the trajectory flowing from continuous on-line control when the distance between the moving hand and the target is small, two different forms of intermittent control have been mooted (i) one or more corrective sub-movements superimposed on and overlapping with the original one in the presence of large target displacements (Flash and Henis, 1991; Soechting and Lacquaniti, 1983), and (ii) interruption of the original movement and its substitution with a movement to the new target location with still larger displacements (Georgopoulos et al., 1981). In suppression tasks, this last mode predicts that the original motor program is truncated (suppressed) and replaced by a new program directed to stop the movement. In subsequent sections, we will address the issue of whether processes similar to one or the other of these 3 modes of correction operate at higher levels in the PFS, at least at the cortical output level.

\subsection{Models of on-line corrections}

Different types of generative models have been proposed to account for on-line corrections. One such model assumes that the hand follows a planned minimum-jerk trajectory, and when the target jumps away, another minimum-jerk trajectory connecting the original and displaced target positions is added vectorially to the original plan (Flash and Henis, 1991). A related model (Hoff and Arbib, 1993) involves feedback-control of the minimum-jerk model.

In the Vector Integration to Endpoint Model (VITE, Bullock and Grossberg, 1988), a difference vector (DV) is computed between representations of the target position vector (TPV) and the hand's present position vector (PPV). The output from the PPV continuously specifies desired hand position. The desired velocity vector (DVV) for the hand is the product of DV and an internally generated Go signal. The Go signal can be used to initiate the movement, scale its overall velocity, and halt movement. The PPV is generated internally by continuously integrating the DVV. The VITE model assigns these computational modules to specific neural networks. Thus, PPV would be encoded in area 5 of PPC. DV would also be computed in area 5 by comparing PPV with a TPV signal fed by the dorsal stream, but for fast reactions signals may also be input to PPC by the subcortical visual pathway passing by the pulvinar. DVV would be computed in M1. The Go signal might be finally provided by basal ganglia and thalamus.

In feedback control based on internal models, a comparator monitors the difference between the current estimated position of the hand (PPV) and its desired position (matching target position, TPV) and velocity (DVV), and this difference signal (DV) is fed downstream to a controller which outputs the correct motor commands (Gomi, 2008; Sabes, 2000). The desired position is estimated by combining sensory information with the output of a forward predictive model, the sensory channels including both vision and proprioception in healthy subjects. However, because a de-afferented patient was able to generate rapid corrections (Bard et al., 1999), proprioceptive information may not always be critical for response initiation.

Classical optimal control minimizes a homogeneous cost (e.g. energy consumption, movement jerk, rate of change of joint torques, endpoint variance), and treats all other task goals as constraints specified externally. Optimal Feedback Control (OFC, Todorov and Jordan, 2002) goes beyond this approach by optimizing composite cost functions. These functions include, but are not limited to, energetic efficiency, endpoint positional accuracy (bias and variance), endpoint stability (bringing the movement to a complete stop), and movement speed. Moreover, the corresponding feedback gains are not constant, but depend on the accuracy requirements of the task and can change during the task 
execution. In particular, OFC predicts that feedbacks should be modulated during a movement depending on the distance to the target. Liu and Todorov (2007) tested this prediction in a DoubleStep protocol. Consistent with the model, in case of target jumps occurring near the start of the movement, there was no change in movement speed and the trajectories progressively converged to the displaced target during the remaining part of the movement. Instead, when the target jump occurred close to the end of the movement, the participants reacted more strongly producing both a change in the movement speed and a lateral movement toward the target. Moreover, in this latter case, participants failed to fully compensate for the target displacement. Prima facie, it appears surprising that the optimal solution for late target jumps involves an incomplete correction. However, it turns out that, near the end of the movement, the optimal controller becomes less sensitive to positional errors, and instead aims at stopping the movement in a stable manner (Liu and Todorov, 2007).

Feedback gains have been measured directly during target jumps by Dimitriou et al. (2013). They found that the visuo-motor gain was non-specifically reduced for all target jumps at the time of the jump. Instead, the same gain measured 100 ms later increased for perturbations that increased the distance to the target, and decreased for perturbations, which reduced that distance. These results confirm the flexible adaptation of OFC to task demands, as well as the time-varying nature of the feedback, which allows on-line modifications as a function of task goals.

\subsection{Kinematics of on-line corrections}

Depending on the time interval between the presentation of the first and second target, one observes different gradual transitions in trajectory (Henis and Flash, 1995; Soechting and Lacquaniti, 1983; van Sonderen et al., 1989). Thus, when the interval is longer than about $100 \mathrm{~ms}$ and the initial and the displaced target locations are in different directions with respect to the start position, the hand is first directed toward the initial target and then the trajectory turns toward the displaced target. Instead, when the interval is shorter than about $100 \mathrm{~ms}$, the initial direction of the hand trajectory is intermediate between the directions of the two targets, and becomes progressively closer to the second target as the time between the target displacement and the initial movement onset increases. Patients with Optic Ataxia stemming from neurological impairments of PPC (Gréa et al., 2002, see Fig. 4A) or normal subjects with transient inactivation of this region by means of TMS (Desmurget et al., 1999) do not produce kinematic on-line corrections as effectively as healthy subjects. Accordingly, the subject either ignores the second target all together, or he/she moves to the first target, and only after reaching it, moves to the second target. The impairment is especially pronounced when the target is presented in the visual periphery (which is especially sensitive to movement direction, Paillard, 1982). Human Optic Ataxia and its animal models are dealt with in detail in Section 4.

\subsection{Muscle synergies for on-line corrections}

Most behavioral investigations of on-line corrections of reaching movements have been carried out at the kinematic level (see above). However, some early studies reported the changes in electromyographic (EMG) activity in response to a shift in target location, but the observations were limited to a few arm muscles and movement conditions (Gielen et al., 1984; Megaw, 1974; Soechting and Lacquaniti, 1983). Nevertheless, these studies provided the first hint that corrections may depend on muscle synergies, namely coordinated recruitment patterns of groups of muscles at the shoulder and elbow joints. Muscle synergies effectively address the issue of redundancy that we mentioned at the outset, because by recruiting groups of muscles with synergyspecific muscle activation waveforms, appropriately scaled in amplitude, the CNS reduces the number of parameters to be specified.

The suggestion that on-line corrections may involve muscle synergies similar to those employed in unperturbed reaching has been taken up in a couple of recent studies. In both studies, EMG activity was recorded simultaneously from several muscles. Fautrelle et al. (2010) used a simple method to correlate the latencies of muscle activities for a target jump to a single location. They found that both initiation times and correction times were strongly correlated for some pairs of muscles, independently of their occurrences during the motor sequence and independently of the location of the muscles at the anatomical level.

d'Avella et al. (2011) applied modern factorization techniques to the EMG activity of 16 muscles of the upper limb in a protocol involving abrupt changes in target location at different delays during fast reaching movements in multiple directions in a frontal plane. They tested the hypothesis that the same time-varying muscle synergies, which are used for reaching movements to fixed targets, are also used for the on-line corrections required by target jumps. To this end, time-varying muscle synergies were first computed from the phasic normalized EMGs of unperturbed reaching movements to the same targets. Three such synergies explained about $80 \%$ of the data variance in agreement with previous results (d'Avella et al., 2006, 2008). Strikingly, the superposition and modulation in amplitude and timing of the same three synergies was able to account also for the EMGs of movements involving a target shift.

We remarked in a previous section that, at the kinematic level, there is evidence for the superposition of the hand trajectory from the starting position to the initial target with that of the trajectory, appropriately delayed, from the initial to the final target (Flash and Henis, 1991). However, at the muscle level, d'Avella et al. (2011) found that the muscle patterns underlying reaching movements to displaced targets were not accurately described by the delayed superposition of the corresponding point-to-point patterns, even after amplitude modulation. In order to adequately fit the EMG patterns they obtained, d'Avella et al. found it necessary to modulate in amplitude and timing the recruitment of the point-to-point timevarying muscle synergies employed in the corresponding radial and tangential point-to-point movements.

Time-varying muscle synergies may thus provide a set of a few basic patterned modules for the performance of different conditions of a reaching task. Specific instantiations of the commands require the specification of the recruitment amplitude and onset time for each synergy. As mentioned in a previous section, an internal model of the dynamics of the musculoskeletal system can be exploited in Optimal Feedback Control (Todorov and Jordan, 2002). However, this model is fairly complex and context-dependent and it might be difficult to acquire by the CNS. Muscle synergies offer a viable alternative solution to complex internal models. They may provide the basis functions that allow the acquisition and use of a simple mapping from task goals and initial states into motor commands (d'Avella et al., 2006). This mapping is efficient because it relies on a reduced number of parameters that need to be adjusted, stored, and retrieved.

Notice that, while the muscle activity for reaching to a fixed target can be largely pre-programmed when the accuracy demands are low, the muscle activity in response to a change in target location requires adjustments driven by visual and proprioceptive information. Therefore, a feedforward controller based on muscle synergies must be complemented by a feedback for on-line corrections. The results of d'Avella et al. (2011) suggest that a common modular architecture based on patterned motor commands is used for both the control of unperturbed reaching movements 
and for their visually guided on-line corrections required by target jumps. These corrections may be planned as discrete corrective movements from the initial to the final target locations to be superimposed to the initial movements, once the target change has been detected. They may be implemented by the superposition of a few time-varying muscle synergies, after adjusting the synergy recruitment parameters associated with the corresponding corrective point-to-point movement; this would enable the system to accommodate different initial states of the musculoskeletal system (d'Avella et al., 2011).

These results are compatible with a synergy-based intermittent controller. In a synergistic controller, intermittent corrections would be implemented by re-using the mapping of goals and states into the synergy recruitment coefficients (d'Avella and Lacquaniti, 2013). Sensory feedback would still be processed continuously to update the estimates of the current state and goals (target location). The update is necessary to prepare the synergy coefficients for the appropriate corrections. In addition, sensory feedback might be used to compute an error signal triggering a correction by recruiting a set of time-varying synergies. The trigger could be based on threshold processes mentioned above. Because each synergy has a fixed duration, different synergies or multiple instances of the same synergy may partially overlap, thus resulting in a smooth movement that would superficially appear as continuously controlled.

However, intermittent controllers are not the only ones compatible with a synergistic implementation. An alternative solution is represented by a synergy control that allows on-line changes of the temporal structure of the muscle patterns based on sensory feedback. The group of muscles coupled by the synergy would remain fixed, but the temporal pattern of activation would be shaped by delayed feedback signals from the periphery. Evidence for this control scheme has been obtained in studies of postural responses to perturbations (Safavynia and Ting, 2013).

\subsection{Temporal coupling between eye and hand movement during on-line corrections}

An important aspect of on-line control of reaching concerns the degree of coupling between the gaze and hand motor systems during fast corrections. This is a special aspect of the general problem of eye-hand coordination, which has recently been reviewed by Crawford et al. (2011). In the temporal domain, the study of the correlation between the eye and hand latencies taken to initiate a movement under different conditions has revealed a certain interdependency between the two systems. The reported degree of correlation varies not only across experiments (Gielen et al., 1984; Sailer et al., 2000), but can also be found among studies using similar protocols. Thus, Sailer et al. (2000) found a higher correlation between eye and hand reaction-times (RTs) in tasks requiring an intentional correction of combined eye and hand movements, than in tasks involving reflexive movements, such as those evoked in the anti-saccade protocols, where subjects are required to move both the eye and the hand in an opposite directions with respect to a visual target. A tight association between the time of initiation of eye and hand movement has been found during simple "look and point" paradigms and consisted in strong linear correlation between hand RT and eye RT (Herman et al., 1981). In other similar tasks, Gielen et al. (1984) found a significant correlation between eye and hand RTs, while Prablanc et al. (1979) had argued that eye and hand latencies are in general "poorly" correlated. However, no $p$-value associated to the Pearson's coefficients was reported for this latter study.

Similar measurements were also performed in studies where reaching movements were performed under an unexpected target displacement. Under this condition a tight correlation was observed between the corrective hand movement and the time of the second saccade (Neggers and Bekkering, 2002). When compared to the single-step reaches (Gielen et al., 1984), the correlation between eye and hand RT to initiate the hand movement remained similar if the target jump was in the same direction as the first target. When a target displacement in opposite directions was tested at different times, the correlation was not influenced by the displacement only if this occurred beyond $125 \mathrm{~ms}$ after presentation of the first target. Faster target displacements resulted in significantly smaller correlations between eye and hand reaction times. In this study, however, no correlation has been reported between the time of the second saccade and that of hand movement correction.

Correlations in eye and hand RTs might be due to a common neural mechanism for eye and hand movement control, which seems to be plausible not only on the basis of the behavioral evidences of eye-hand coupling described above, but also on the basis of neurophysiological literature, that offers examples of interdependencies between eye and hand control systems. As an example, the analysis of the temporal evolution of population activity in the PPC showed the existence of a common, simultaneous activation (BattagliaMayer et al., 2007) during different visuomotor tasks involving eye and hand movement. This activation was effector-independent and might reflect a neural mechanism that provides a common input drive to the eye and hand control systems, for planning, execution and online adjustments. This central command system can be tuned to the individual effectors control centers downstream in the CNS. This idea would be also in line with the consequences of transient inactivation of PPC prior to the onset of saccadic eye movements, that results in the disruption of natural correlation between eye and hand movement amplitude during reaching (van Donkelaar et al., 2000).

\subsection{Motor decision}

The correct execution of sensory-guided motor acts requires preliminary perceptual discrimination. The related literature is out of the scope of the present paper and has been previously reviewed (Gold and Shadlen, 2007; Heekeren et al., 2011). Here we present factors influencing motor plan development and correction, occurring when perceptual decision processes are completed. Even in very simple motor tasks, the time of movement onset is highly unpredictable. As discussed above, many factors can influence a response (reaction) time and the wide range of RT durations which are typically found suggests that a complex mechanism underlies motor decision. This is illustrated by the findings on the countermanding task (Logan and Cowan, 1984): on some proportion of trials of this reaction time task, a second signal occurs upon which the subject must abort the intended response. In an influential paper, Logan and Cowan (1984) put forward a race model of the task, the control of motor response (following Go signal presentation) and motor suppression (consequent to Stop signal) being viewed as the outcome of a dynamic race between two independent processes running toward a common threshold. According to the model, when a Stop signal is presented during the RT, the probability of successfully suppressing the programmed movement depends on the relative speed of the Go and Stop processes.

A modified version of the race model including a further Go process, starting at the time of appearance of the second target (Camalier et al., 2007), has been used to successfully explain the eye behavior in the saccadic Double-Step task. Similarly, an interactive race model where Go and Stop processes are viewed as nonindependent (Boucher et al., 2007) explains, more efficiently than the classic model, the process of saccade generation in the countermanding task. However, in the remaining part of the manuscript we will refer to the original race model where the two processes are 
considered separated, when not otherwise specified, since most of the experiments in the literature have been designed accordingly (see also Verbruggen and Logan, 2009).

An important and often neglected aspect of the race model and of the behavioral evidence relevant to it, is that movement preparation is dissociated from movement execution in a fashion analogous to that obtained with the different forms of the delayed response task (Wise, 1985). In successfully canceled Stop trials, motor action is prepared but never transformed into a movement; in complementary fashion, the motor plan is mature well in advance of motor execution in the Delay task. In both cases movement preparation could be thought of as a sub-threshold representation. However, all attempts to demonstrate the existence of a motor plan at an advanced level prior to its realization, which could then be realized by sub-threshold activation-based control, have been inconclusive for reaching movements, although evidence exists for analogous neural modulations with respect to saccadic eye movement control (Schall, 2001; Shenoy et al., 2013). Recent neurophysiological evidence and complementary simulations suggest an alternative, namely that the cortical control of movement generation resides in the dynamic of motor neurons under attractor-based control (Mattia et al., 2013; Shenoy et al., 2013). In this last scenario, mature motor plans corresponds to rapid and hierarchically organized changes in the state of neural activity occurring after target presentation. Different modules with preferred high- and lowfiring states, and with heterogeneity in excitability (Mattia et al., 2013), are gradually recruited and could evolve in the corresponding motor act when external control breaks are released. Thus, motor plans can be assumed to be primarily rooted in premotor cortex, but motor execution would also involve other frontal structures with a cognitive control function, including the pre-SMA/SMA complex or basal ganglia.

\subsection{Summary}

In this section, we considered the complex nature of the computational steps involved in reaching toward a target, whether stationary or jumping. We argued that the integration of visual and proprioceptive signals in multiple reference frames affords most efficient localization of the stimuli, weithing noisier sensory signals less. Conspicuous sensorimotor delays appear to be partially compensated at several different stages in the nervous system, also by taking advantage of internal models which mimic both the target and arm dynamics. We contrasted two different control modes for correction or suppression of movements, i.e. continuous versus intermittent control, each one offering specific benefits in the appropriate context. Thus, when the distance between the hand and the target is small, continuous on-line control generates smooth modifications of the trajectory. Instead, in the presence of large target displacements, intermittent control generates one or more corrective sub-movements superimposed on and overlapping with the original one. Finally, when target shifts are even larger, the original movement is interrupted and replaced by a new movement. At the execution stage, on-line corrections appear to involve muscle synergies similar to those employed in unperturbed reaching. However, while the muscle synergies for reaching to a fixed target can be largely pre-programmed, the muscle synergies in response to a change in target location require adjustments driven by visual and proprioceptive information. The strong degree of hand-gaze coupling during on line correction shown by behavioral studies has been discussed in relation to the experimental neurophysiological evidence of a potential common and effectorindependent parietal command mechanism. Finally, we considered the mechanisms underlying motor decisions, dwelling especially on the race model.

\section{Neurophysiological studies}

\subsection{Cell-recordings in the monkey during on-line corrections}

In spite of the wealth of information available on on-line control of movement from behavioral studies, only four cell-recording studies exist in the literature on the on-line control of hand movement trajectory (Archambault et al., 2009, 2011; Dickey et al., 2013; Georgopoulos et al., 1983). They have been devoted to the analysis of the role of premotor, motor, and posterior parietal cortex. In the first three studies monkeys were trained in a Double-Step centerout task, where they make planar arm movements to targets located at $45^{\circ}$ apart along the circumference of a circle (Georgopoulos et al., 1983) or natural 3-D reaches from a center position to targets presented at the vertices of an imaginary cube (Archambault et al., $2009,2011)$. After the presentation of the first target, a second target appeared at $90^{\circ}$ or at $180^{\circ}$, either during the RT or at the onset of hand movement to the first target, so that the target appeared to "jump" from one position to another. Under such large target displacements, the hand initially moved toward the first target and then changed direction toward the second one, with a velocity profile characterized by the presence of two peaks. When the second target was presented during the hand RT to the first one, the RT of corrected reaches was about the same of the unperturbed ones (Georgopoulos et al., 1983; Archambault et al., 2009, 2011). Thus, a "Psychological Refractory Period" (Welford, 1952) does not seem to occur when two movements are combined one into the other. Therefore the need to respond to a target "jump" does not influence the duration of movement preparation.

At the neural level, in motor (M1/area 4), dorsal premotor (PMd/F2, area 6) and posterior parietal (PE/PEc, area 5) cortex the modulation of cell activity during both direct and corrected reaches predicts with a high degree of fidelity the modification of the hand trajectory (Fig. 1A), with a better prediction, however, made by parietal than by frontal neurons. Interestingly, a drop in the correlation between hand kinematics and neural activity occurs when the movement trajectory is corrected (Fig. 1B), probably due to the interference or coexistence between the old and the new motor plan. This drop is less pronounced for posterior parietal cortex (PPC) than for premotor and motor cortex, and supports the assumption of a central role of parietal cortex in the trajectory state estimation. The activity of most cells takes place before the change in hand kinematics and this occurs earlier in motor than in premotor and PPC (Fig. 1C), in line with the role of motor cortex in the generation of the motor output.

Neural activity related to switching motor plans has been described by a recent study (Pastor-Bernier et al., 2012), which also suggests that in premotor cortex a unique biased competition model can account for both initial decisions and for changing motor intention. This account of substitution of motor plans in premotor cortex is related to the above-discussed versions of the race model.

The analysis of hand kinematics during trajectory correction reveals the existence of a high correlation between the hand speed profiles of the Double-Step reach and the two corresponding profiles of single step reaches into which the corrective movement can be decomposed. Thus, when the hand correction is of $180^{\circ}$, the pattern of neural activity observed in M1, PMd, and PPC during corrected reaches can be predicted from that typical of uncorrected ones, by splicing together the two spike density functions corresponding to the single-step reaches, with a delay calculated from matching the relative speed profiles (Fig. 2) (Archambault et al., 2009, 2011; Georgopoulos et al., 1983). In fact, if during the hand movement to the first target, a second target appears in the opposite direction (at $180^{\circ}$ ), the activity pattern associated to the former is somehow silenced, probably due to the early signaling occurring in PMd, and substituted by that observed when the monkey reached 
A

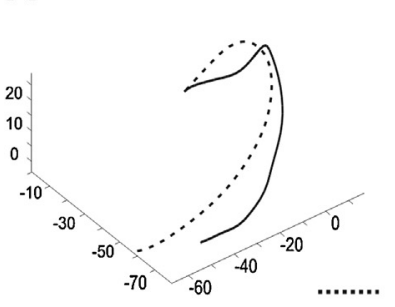

B

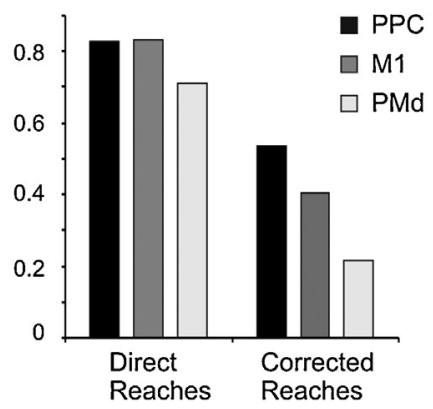

M1

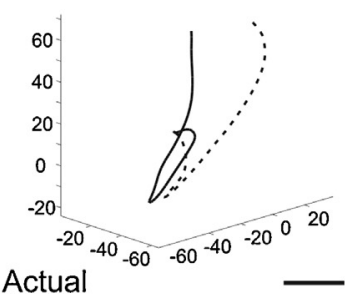

PPC

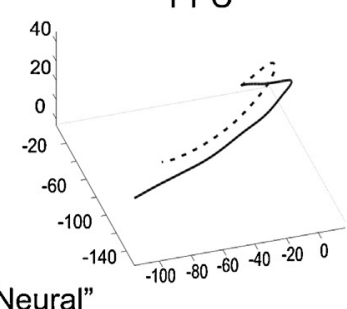

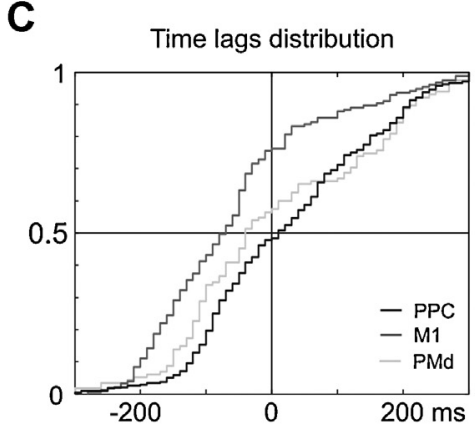

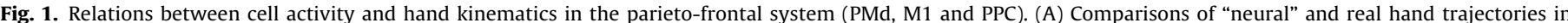

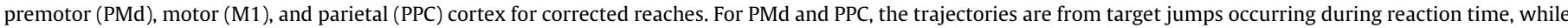

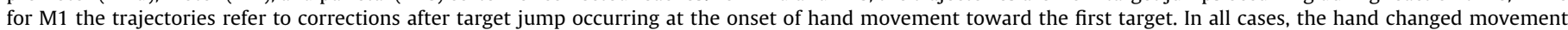

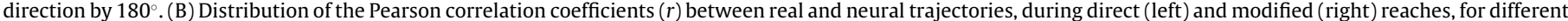

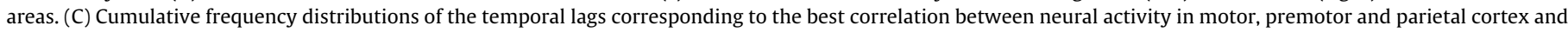
kinematics variables of a multiple linear regression analysis.

Source: Modified from Archambault et al. (2011).

directly to the $180^{\circ}$ target from the initial position, as in the single step reach task. There is a good match between the distribution of the correlation values in the predicted and actual neural activity profiles.

These findings suggest that there exists in the cerebral cortex the same basic neural mechanism, which is used both for trajectory formation and correction. Such a mechanism would be rooted in the graded and time-varying utilization of available kinematic variables within the same neural assembly, rather than in the recruitment of a selected neuronal population, which would have an activity specifically tuned to the movement correction. In fact, in PPC, PMd and M1, the same neurons are active during both direct and corrected reaches, although with different timing and kinematic relationships. No evidence was found for a specific population of neurons active only during corrective movements (Archambault et al., 2009, 2011).

That the same cortical cells tend to be used for initial trajectory formation and correction provides a picture highly consistent with that offered by the behavioral observations on the kinematics and on the pattern of muscle activity discussed in a previous section of the manuscript (d'Avella et al., 2011). Changing a motor plan requires the modulation in amplitude and timing of the recruitment of the same muscle synergies used for direct, unperturbed movements. Thus the simple organization outlined in Scheme 1 seems sufficient to account for the neurophysiological evidence on reaching corrections.

A more recent study has explicitly addressed alternative movement correction schemes at the neural level (Dickey et al., 2013). Monkeys acted on a manipulandum so as to move a cursor on a screen toward visual targets that changed location in space, thus requiring online adjustments of cursor trajectory. Under such conditions, the activity of the majority of cells in motor and premotor cortex during corrective movements was fitted by the same linear model adopted for unperturbed reaches, while the activity of one third of the cells appeared to obey a substitution scheme, in which the corrective movement could be reconstructed from its component parts; these findings are therefore compatible with an intermittent, discrete control mechanism. This study differs from those of Georgopoulos et al. (1983) and Archambault et al. (2009, 2011) not only because it required movement at a single joint, but also because the second target was always presented after the onset of movement toward the first one, thus requiring a late correction of an already matured motor plan. Furthermore, the task required a complex visuomotor transformation, due to the dissociation between the spatial position of the targets on the screen and the manipulandum.

\subsection{Timing of cell activity during correction signaling}

An interesting question is when during on-line control of hand movement the upcoming change of hand trajectory is signaled in the parieto-frontal system. When the second target is presented during the hand reaction-time (Fig. 3, left panels), the population activity in premotor, motor and parietal cortex signals the change of movement trajectory before or just around the time when the hand starts moving toward the first target, with earlier signaling by PMd and a later one by PPC, occurring just after movement onset. The same time relationships across areas are maintained when the target shift occurs at the onset of hand movement (Fig. 3, right panels), although in this case the earliest signaling of trajectory correction occurs during the hand movement toward the first target.

The analysis of the timing of activation of the population in premotor, motor and parietal cortex indicates that signaling of both movement initiation and correction occurs first in premotor cortex, while the later activation of parietal cortex probably reflects the specification of the trajectory to be implemented by M1 (Fig. 3), as illustrated in Scheme 1. The earlier activation in PMd can be explained as a signal to change the original motor plan once the new 

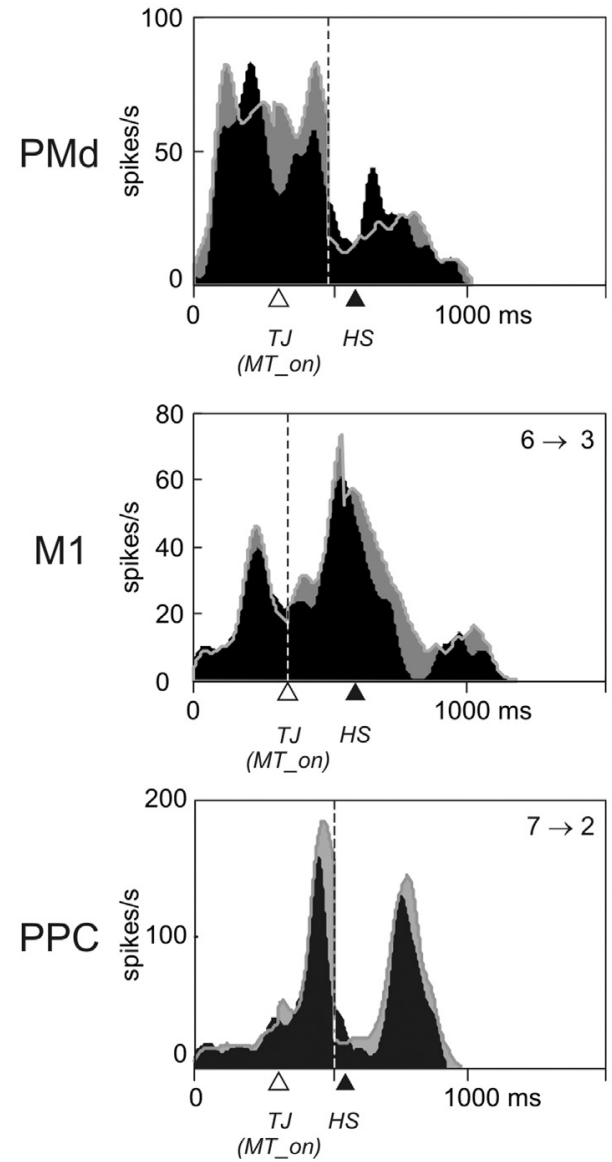

Fig. 2. Predicting cell activity during trajectory correction from the activity associated to the single-step direct reaches in the parieto-frontal network (PMd, M1 and PPC). Overlap of single cell activity observed during hand movement correction (Double-Step, black SDF) of $180^{\circ}$ with that obtained by combining, tip-to-tail the two spike density functions (gray) associated with direct reach from the center toward the first target location and from the center to the second (final) target position. The vertical dashed line represents the time of truncation of cell activity for the first direct reaching movement. The time scale is aligned to the time of first target presentation, the white triangle indicated the time of target jump (TJ), while the black triangle indicates the time of hand trajectory shift (HS). MTon indicates onset movement-time.

Source: Modified from Archambault et al. (2009, 2011).

target location has been detected Altogether, the available results lead to the hypothesis that PMd provides a higher-order control signal to update movement under changes of the visual scene, such as when a visual target jumps from one position to another. In general, the PMd system is held to be required when a new motor plan is initiated or its goal changed in a discrete/intermittent fashion. In a later section we will argue that the same system comes into play when a motor plan must be suppressed. In fact, the measured neural latency in response to the target jump is comparable to what has been reported in the same part of PMd using the Stop task (Mirabella et al., 2011).

One can also speculate that PPC plays a pivotal role in implementing the transformation entailed by the new motor command, through computing the required new trajectory. This would be based on the fine-grain encoding of limb kinematics in this area. Finally, M1 is held to play a direct role in providing precise control of the implementation of the motor plan trajectory on an ongoing basis. Hardly surprisingly it appears to be directly influenced by PMd as well as via PPC (see Scheme 1), as indicated by the relative times in different structures at which the activity in corrected reaches diverges from the uncorrected one (Fig. 3).

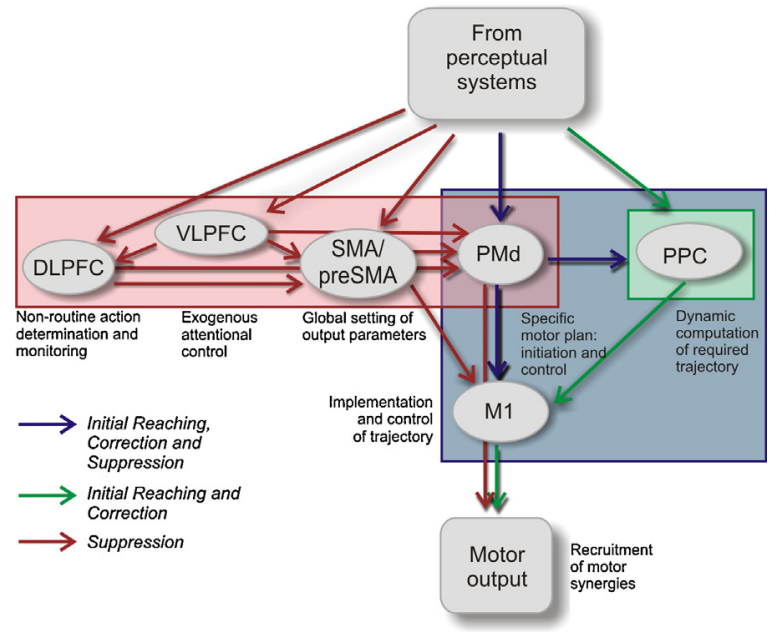

Scheme 1. Simplified flow diagram of processes undertaken by the key cortical systems involved in initial standard reaches or correction or suppression of reaching movements. The direction of arrows is not meant to indicate detailed anatomical connectivity, but rather the predominant flow of information (see Section 3). Transmission of information between pairs of systems is sufficiently fast to allow bidirectional interaction and recursive signaling in reaching the appropriate output of the pair. For simplicity the systems involved in the two suppression tasks discussed - Go/No-Go and Stop tasks - are conflated; the principal difference lies in the processes that involve the pre-SMA (see Section 5). More specifically, blue arrows indicate information transmission used in initial reaching, its correction and its suppression. Green arrows indicate information transmission used for initia reaches and correction. Red arrows indicate information transmission used only for suppression. The colored boxes cover the systems involved in initial reaches, correction and suppression (blue), initial reaching and correction (green), and reaching suppression (red). "Perceptual systems" include both dorsal (object location) and ventral (object properties and identity) streams. "Non-routine action determination" refers to the high-level processes occurring when, say, an unusual strategy is adopted, such as slowing down responding in the Stop signal paradigm to make it easier to inhibit, if the Stop signal does occur. "Monitoring" refers to the complementary high-level process of being set or prepared to interrupt on-going behavior should a Stop signal occur and so speeding inhibition of the on-going response (see Coxon et al., 2006; Gentet, 2012; Shallice and Cooper, 2011, chapter 9 for discussion of these two processes). "Exogenous attentional control" refers to the actua process of allowing stimulus-driven interruption of central processing to take place (see Corbetta and Shulman, 2002). DLPFC, dorsolateral prefrontal cortex; VLPFC, ventrolateral prefrontal cortex; M1, primary motor cortex; pre-SMA/SMA, pre- and supplementary motor cortex; PMd, dorsal premotor cortex; PPC, posterior parietal cortex.

\subsection{Potential neural mechanisms for movement correction}

The pattern of the population activities in PMd, M1, and PPC typical of direct and corrected reaches provides clues as to the potential mechanisms through which movement correction might be achieved. When the target jumps during RT (Fig. 3, left panels), the overall similarity of the shape of the temporal evolution of the population activity associated with direct (gray) and corrected (black) reaches supports a model based on a continuous adjustments from target jump to final target position. In contrast, in each area the sharper divergence observed in the case of target jump at the onset of hand movement (Fig. 3, right panels), when the time interval between first and second target presentation is large, rather suggests a discrete substitution scheme (see also Georgopoulos et al., 1983), probably based on intermittent control. However, it must be stressed that single cell activity in both frontal and parietal areas seems compatible with both continuous and intermittent control and that the predominance of one coding scheme over the other across different parietal and frontal areas remains a challenging subject for future research.

The change or suppression of an original motor plan can be implemented in different ways. Since cortico-cortical fibers are generally excitatory (Conti et al., 1988), premotor projections to 


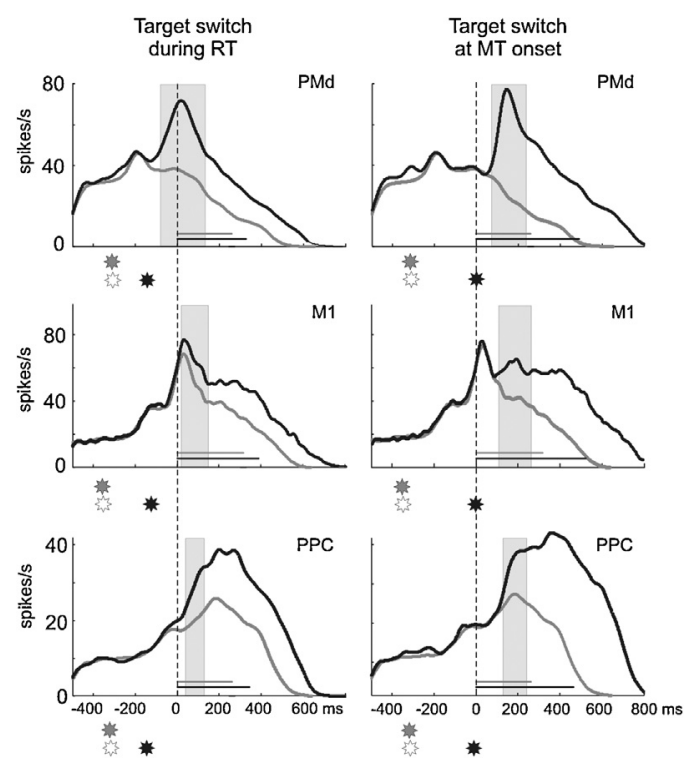

Fig. 3. Each panel shows the comparison of the population spike density functions (pop-SDFs) of the neural activity recorded in frontal (PMd, M1) and parietal (PPC) cortex during direct reaches (gray curve) versus corrected ones (black curve), when the target jumped during RT or at the onset of MT. During on-line corrections, the pop-SFDs were obtained by combining single-cell activity first directed toward the target opposite to the preferred direction (anti-PD) and then to the PD. This activity is compared to direct reaches toward the first target (anti-PD) to show the time at which the two population activities diverge. The stars indicate the time of target presentation during direct (gray) and corrected reaches (white, first target; black, second target), while the horizontal bars indicate the mean duration of hand movement time in the two conditions (gray, direct reaches; black, corrected reaches). The time scale is aligned to the onset of hand movement (dashed line at $0 \mathrm{~ms}$ ). In each graph, the vertical gray rectangle includes the time spanning from the moment in which neural activity associated to corrected reaches significantly diverges from that of the direct reaches, to the time of change of hand trajectory.

Source: Modified from Archambault et al. (2011).

interneurons which are inhibitory on M1 cortico-spinal neurons, and/or gating of the descending motor command at the level of spinal interneurons through direct premotor projections to the spinal cord (Dum and Strick, 1991) can provide the anatomical substrate for movement updating through a direct influence on the original motor plan or ongoing movement. Alternatively, the articulation of a new trajectory in PPC might modulate the input to $\mathrm{M} 1$, and thus lead to the modification of the original action, without requiring active suppression of the original planned movement. This additional mechanism can operate on a fine time scale and in a very fast way, if one considers that for parietal area 5 (PE/PEc) the estimated average conduction delays are about $2 \mathrm{~ms}$ with M1 and 3.5 ms with PMd (Innocenti et al., 2013). The modulation in the time of this process can lead to a change of an ongoing movement and its substitution with a new one, when the time between the first and second target presentation is long and the correction required is large, compatible with an intermittent control mechanism. By contrast, if the interval between first and second target presentation is short and the first movement is just being born, then this can be continuously changed into a new one. The question then arises as to how these potential alternative ways of movement correction can be implemented. One possibility is by exploiting the potential inherent to the large spectrum of conduction delays through which cortical areas communicate with each other and with subcortical structures (Caminiti et al., 2009; Innocenti et al., 2013; Tomasi et al., 2012), thanks to the spectrum of axon diameters characterizing cortico-cortical projections, as well as cortical projections addressed to basal ganglia and spinal cord.
It can be hypothesized that populations of premotor neurons with a similar restricted spectrum of large axon diameters, and therefore fast conduction velocities, will be recruited so as to suppress the discharge of cortico-spinal neurons and/or of spinal interneurons, and therefore an ongoing movement, through inhibitory cortical and/or spinal interneurons. The existence of premotor and/or parietal cortical neurons with a wide spectrum of small axon diameters and long conduction delays could favor a smooth and continuous transition process over a large time-scale, thus allowing the progressive substitution of an old movement with a new one. This mechanism can benefits from the large spectrum of axon diameters, therefore of conduction delays, of descending fibers from motor cortex (Innocenti et al., 2013; Lemon, 2008).

Therefore, the same distributed system, although with different complementary networks, can subserve different types of corrections, as well as suppression of reaches, if one considers the advantages inherent the utilization of the spectrum of conduction delays available for communication between the connecting edges of the network.

\subsection{Activation and silencing studies in humans}

In humans the cortical network involved in on-line control of hand movement has been studied using both Transcranial Magnetic Stimulation (TMS) and Functional Magnetic Resonance Imaging (fMRI). When parietal neural activity is temporarily disrupted through TMS delivered at the onset of hand movement, normal subjects are able to make direct reaches, but fail to make on-line corrections when the target moves in space (Della Maggiore et al., 2004; Desmurget et al., 1999). Since inactivation or lesioning of parietal cortex can result in impaired estimates of limb position (Wolpert et al., 1998), it has been speculated that the defective online correction is a consequence of an erroneous computation of the motor error. This is in line with recent neurophysiological studies of parietal area 5 in monkeys (Ferraina et al., 2009).

The involvement of parietal cortex in on-line control of hand movement in humans is also supported by a recent fMRI study (Reichenbach et al., 2011) which, during correction trials, shows an activation cluster centered on the anterior part of the left intraparietal sulcus (aIPS), and also involving the anterior part of the supramarginal gyrus (aSMG), and of the SPL. A smaller activation cluster was also observed at group level in a roughly corresponding region of the right aIPS. The subsequent TMS stimulation on the position of the activated cluster, resulted in impairments in the timing, but not the accuracy, of corrective movements only when the left hemisphere was silenced. This cluster was in a different location from that stimulated by Desmurget et al. (1999). This study does not report whether the deficit refers to hand reaction and/or movement time. The activation of a cluster in the right parietal cortex could be related to the attentional shift associated to the correction of movement, given the involvement of the right SMG in attention (Perry and Zeki, 2000). However, it is important to stress that in the study of Reichenbach et al. (2011) subjects moved a joystick attached to the index finger to visual targets. This is substantially different from hand reaching, since it does not involve the same type of spatial transformation, kinematic constrains and pattern of muscle activity. Furthermore, in this study the behavioral task used during TMS stimulation was performed in a virtual reality environment and was therefore different from that used during the fMRI experiment. This study therefore does not undercut the conclusion from monkey neurophysiology studies that there are no systems in parietal cortex specifically concerned with movement correction.

At the same time, activation of the intraparietal region of the left hemisphere and of the SPL during on-line hand adjustment was shown in earlier imaging studies (Clower et al., 1996; Desmurget 
et al., 2001) using Positron Emission Tomography (PET), which, however, has poorer spatial resolution than that of fMRI.

TMS studies indicate that another area participating in on-line correction of hand movements is dorsal premotor cortex (PMd), but only when trajectory correction depends on visual cues, such as vision of the hand during a visuo-motor adaptation task (Lee and van Donkelaar, 2006). When subjects are prevented from seeing their hand, TMS does not disrupt the ability to make on-line adjustments, as it does when vision of the hand is available. This keeps in line with the observation from cell recordings in monkeys, indicating that as soon as the visual target displacement is detected, the earliest signaling for on-line correction within the parieto-frontal network occurs in PMd, as compared to M1 and area 5.

Further studies based on a rigorous quantitative control of both behavior and brain activation and more naturalistic experimental set-ups will be required to uncover the whole cortical network underlying on-line control of reaching in humans. In particular it should be noted that reaches are characterized by a complex kinematic and by the recruitment of time-varying muscle synergies which are modulated in amplitude and timing, and this differs substantially from tasks where more elementary hand movement are required.

\subsection{Summary}

In premotor, motor and posterior parietal cortex there exist significant relationships between neural activity and hand kinematics (position, speed and movement direction) during both direct unperturbed reaches and corrected ones. When an original motor plan is changed, the neural activity profile typical of the movement to the first target smoothly evolves into that associated to the movement toward the second target, as observed during direct reaches. No population of cells have been found as selectively recruited only during online adjustments. Therefore, these can only be based on a graded and time-varying utilization of the kinematics variables encoded by neural activity in different areas. During online corrections, parietal cells remain a more accurate predictor of hand trajectory than frontal ones. The time lags of neural activity with hand kinematics indicate that motor, premotor and parietal cortex are activated sequentially, although a large overlap in the timing of their recruitment occurs. After the first target's presentation and its change in location, the population activity in frontal and parietal cortex signals the change of motor plan before the hand moves to the initial target's position. This signaling occurs earlier in premotor than in motor and parietal cortex. It is suggested that premotor cortex encodes the higher-order command for the correction of motor plan as soon as the change in target location is detected, while parietal cortex is responsible for estimating the kinematics of the motor periphery, an essential step to allow motor cortex to modify and control hand trajectory on an ongoing basis. In conclusion, single cell studies in behaving monkeys indicate that the parieto-frontal system can update an original and not-yet-accomplished motor plan during its execution. Both activation (fMRI) and perturbation (TMS) studies in humans point to a crucial role of posterior parietal cortex in online control of hand movement and of premotor cortex when movement correction critically depends on visual monitoring of hand motion.

\section{Neuropsychological studies}

\subsection{Reaching disorders and on-line fast movement corrections in Optic Ataxia}

On-line automatic correction of a movement has been most studied from a neuropsychological perspective in Optic Ataxia (OA).
Patients with OA have impairments in the visuomotor domain, especially when they are required to perform reaching and pointing movements to a target in the periphery (extrafoveal condition), although cases of foveal OA have been observed (e.g. Buxbaum and Coslett, 1998). Reaching errors in such patients can be independent of any primary motor, sensory, praxis or attentional deficit (Perenin and Vighetto, 1988). These patients also tend to make more errors in the visual field contralateral to the lesion (visual field effect) and/or using the hand contralateral to the lesion (hand effect) with a combination of the two being frequently observed (e.g. Blangero et al., 2008).

There has been considerable controversy over the localization of OA in recent years. In OA patients with vascular disease, the lesions tend to be large; thus Pisella et al. (2009) provided the lesion overlap of 11 OA patients, which involved a large area of damage to the occipital and posterior parietal cortices, mainly involving the parieto-occipital junction (POJ), precuneus and IPS, bilaterally. In two series of tumor patients, tested just post operatively, the critical area was similar if slightly more superior in its lower limit in one of the studies (Buiatti et al., 2013; Shallice et al., 2010). Traditionally the syndrome was held to occur following damage to the posterior parietal cortex (PPC) and in particular with superior parietal lesions (see Battaglia-Mayer et al., 2012, for reviews). However, Karnath and Perenin (2005) held instead that the critical area was centered on the parieto-occipital junction (POJ). In their large series, both the inferior parietal lobes and the left superior parietal lobe were involved, and medially the precuneus. However, Coulthard et al. (2006) argued against a critical role for the lateral inferior parietal lobe, pointing to the existence of dissociations between OA and unilateral neglect (e.g. Perenin, 1997), which they held implied that the critical lesion must have only a lateral superior parietal or precuneus localization. In fact, there is a reasonable correspondence between these localizations and those of critical areas found in functional imaging of related tasks in healthy adults. Thus, Blangero et al. (2009) in a meta-analysis focuses on the bilateral POJ, the posterior IPS, and a mid-IPS region as giving rise to reaching impairments.

\subsection{Double-Step paradigm in $O A$}

On the basis of a study in Lyon of an OA patient, IG, inspired by the TMS study of Desmurget et al. (1999) discussed above, Pisella et al. (2000) suggested that a mechanism involved in producing automatic movement corrections for the hand in reaching was also located in the PPC. On $20 \%$ of the trials in this study, a fast correction of the hand trajectory was required, since the target suddenly changed its position at the time of movement onset (perturbed condition). No major abnormal effects were observed on the unperturbed trials. On the perturbed trials, IG mainly produced slow correctional movements, with many fewer fast corrections than controls. Unlike normal controls, she produced no inappropriate correctional movements in the Stop signal condition that was also carried out. Similar findings were also made in a subsequent study of Gréa et al. (2002), where IG again did not adapt the trajectory of the hand in flight, but instead produced two distinct hand movements (Fig. 4A).

From a theoretical perspective, the Lyon group proposed that the impaired performance of OA patients in these tasks could arise from a specific deficit in the on-line visuomotor control involved in making rapid motor adjustments of the hand movement (Pisella et al., 2000; Rossetti et al., 2003). They held that damage to a system responsible for this would also impair pointing toward peripheral targets, since less precise visual information is available and so online adaptation of the movement parameters concerning location is required (Rossetti et al., 2003). This view was developed further by Blangero et al. (2008), who studied another OA patient, CF. CF's 
A

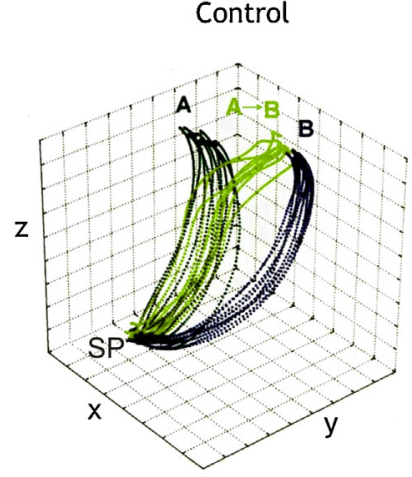

B

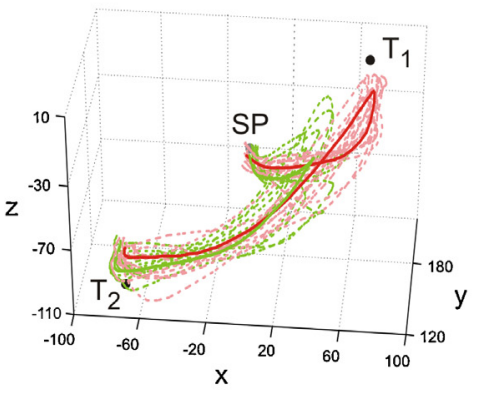

D

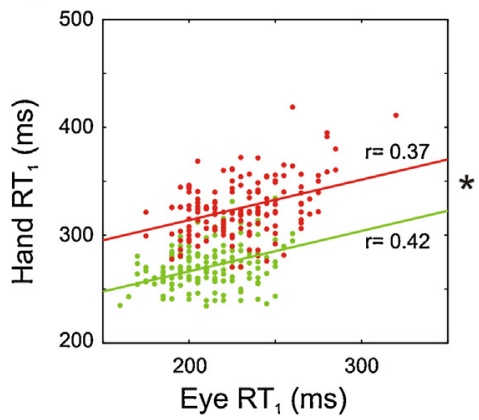

Bilateral Parietal Patient

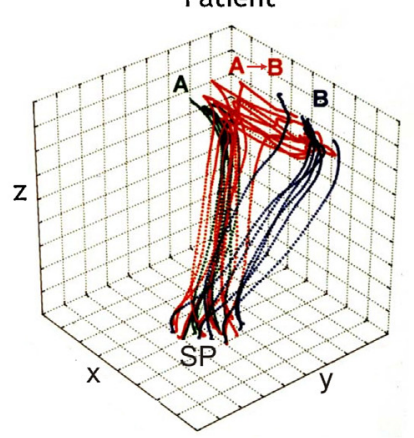

C

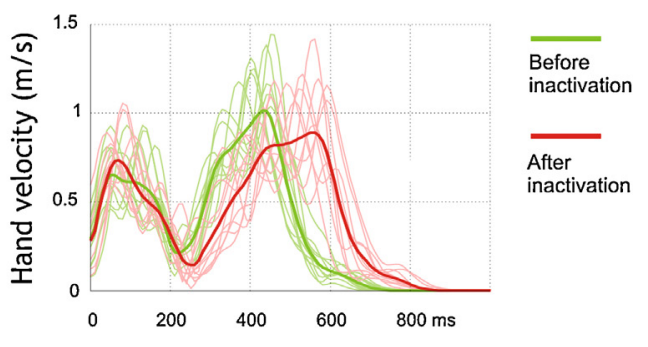

E

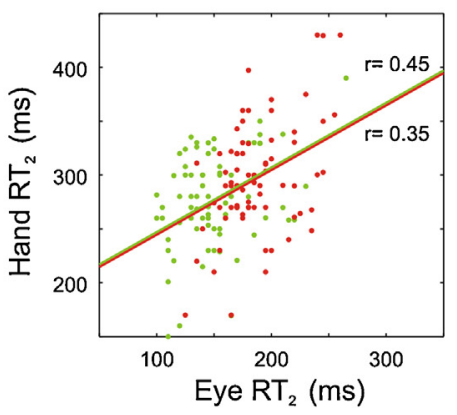

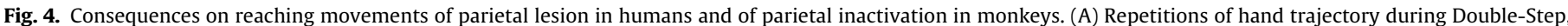

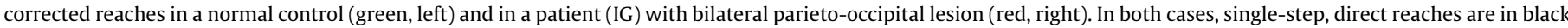

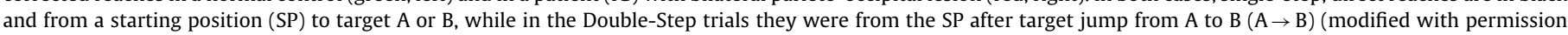

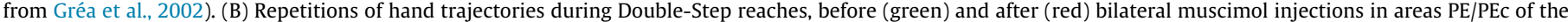

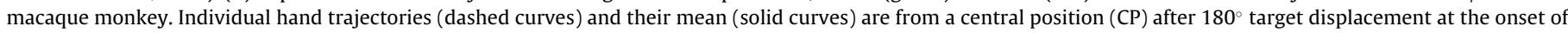

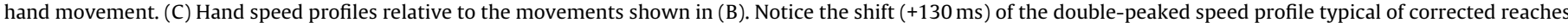

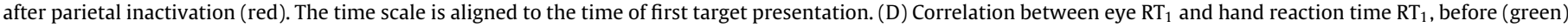

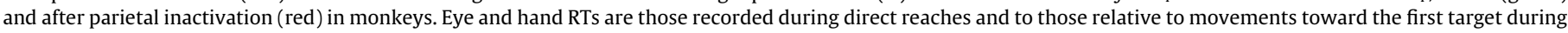

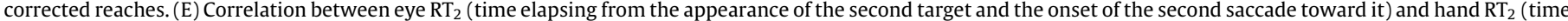

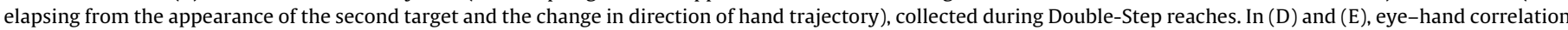

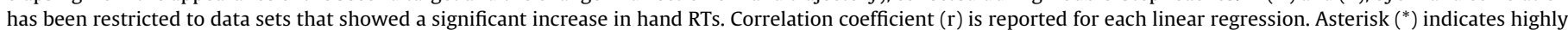
significant differences $(p<0.001)$ between intercepts of 2 linear regressions.

Source: Modified from Battaglia-Mayer et al. (2012).

ability to correct the hand trajectory in flight when a target moves was also impaired as was standard peripheral reaching. Moreover, CF showed the same combination of hand and visual field effects in the two tasks, namely roughly additive impairments with respect to the left visual field, and the left hand. Blangero et al. (2008) therefore put forward a model of the processes involved in visuo-manual transformations, where updating of the reach plan involves two main input stages. The first stage is required for processing of a peripheral visual target but not a foveal one. Damage to such a purely input stage could also give rise to purely perceptual problems; indeed McIntosh et al. (2011) showed correlated disorders of the action and perception of target jump direction in optic ataxic patient, IG. The second stage concerns processing of hand location. Both stages are held to be located in PPC, in the POJ and the MIPS, respectively. These two input stages are then integrated in a third one.

The idea that these two distinct modules are involved is held to receive support from a functional imaging study in normal subjects of Prado et al. (2005). In this study reaching in central vision activated a restricted network, while reaching in peripheral vision activating additional regions including POJ. However, one cannot infer separability of subsystems from one-way dissociations between tasks differing in difficulty (Shallice, 1988).

The model proposed by Blangero and colleagues makes two strong predictions. First, the target representation module is a critical stage both for peripheral pointing and with respect to the size of the shift costs in making Double-Step adjustments. Therefore, the two measures should be correlated positively across patients, By 
contrast, as foveal pointing does not depend on this module, a second prediction is that there should be a much weaker correlation between foveal and peripheral pointing.

However, in a study of 15 patients with parietal lesions, Buiatti et al. (2013) obtained an insignificantly negative correlation $(r=-0.18)$ between the degree of impairment in peripheral reaching for the parietal patients and their shift costs in making an on-line reaching adjustment, with classical dissociations, occurring both ways between impaired and intact peripheral pointing and shift costs. Different systems appear to be involved in the two tasks.

By contrast, Buiatti et al. (2013) found a correlation of $r=0.55$ with respect to the degree of impairment between foveal and peripheral reaching, fitting the idea that essentially the same systems are involved in this pair of tasks. This conclusion meshes with two other findings. In an fMRI study of reaching Martin and Himmelbach (2011) no region was found to be differently activated in peripheral than in central vision. In a TMS study of reaching, Busan et al. (2009) found facilitation of reaction time when TMS was applied over the PPC, but this did not vary with whether the target was central or peripheral.

In contrast the findings fit well with the account of Archambault et al. (2011) discussed in Section 3.1 and the general Scheme 1. On this approach both peripheral and foveal reaching require the same process of trajectory setting to be implemented. Thus the two abilities should correlate positively across parietal patients, as they do. The greater error shown by optic ataxic patients for peripheral targets would occur because of the reduced visual information available to the trajectory-setting system on the location of such targets, as well as for the decoupling between the gaze and the hand signals that seems to have an influence on the emergence of the deficit. On the other hand, on-line correction of reaching requires an additional system that specifies that the trajectory should be reimplemented. Thus double dissociations could theoretically occur between shift costs and peripheral reaching, as they do. In addition, premotor patients were found to have increased shift costs when the target moves, but not to be slower in any other task. This is in accord with the account of Archambault et al. (2011) that the premotor cortex is involved in specifying that a reimplementation of the trajectory is required. That some right parietal patients show increased switch costs and normal peripheral reaching could be explained on the model as resulting from premotor-to-parietal disconnection. However, other parietal systems may also be involved.

\subsection{A non-human primate model of Optic Ataxia}

As illustrated before, patients with OA display a wide elongation of the hand path necessary for reach correction, relative to controls (Fig. 4A). In monkeys, a widely used tool to further elucidate the role of cortical areas is the study of the consequences on behavior of their reversible inactivation. Injection of the GABA-A agonist muscimol in the SPL (areas PE/PEc; Marconi et al., 2001) of monkeys trained to perform direct reaches to visual targets, as well as to correct them when the target moves in space alters both the kinematics (Fig. 4B) and the timing (Fig. 4C) of hand movement during direct and corrected reaches (Battaglia-Mayer et al., 2012). As seen in humans with OA, a longer hand path is observed toward the first target (Fig. 4B), as a consequence of a delayed trajectory correction (Fig. 4C). Under SPL inactivation, both the reaction- and movement time to the first and second target are significantly lengthened. The lengthening of the eye RT was not captured in the study of Hwang et al. (2012), in which the so-called parietal reach region (PRR) was silenced and the monkey tested for direct reaches to targets presented in central and peripheral vision. In this study, the effect of parietal inactivation in the on-line control of eye-hand movement was not assessed.
Concerning eye-hand coordination before and after bilateral SPL inactivation, the hand and the eye RTs to the first target are similarly correlated, so that the increase of the hand RT to the first target can be partially explained by the increase of the eye RT (Fig. 4D). In such a case, parietal silencing, however, yields to a further increase in the hand RT, which cannot be accounted for only by the lengthening of eye RT. Under parietal inactivation, the correlation between the hand and the eye RT to the second target (Fig. 4E) shows that the latter largely accounts for the former. Thus, slowing of the eye movement response significantly contributes to the altered initiation and correction of hand movement typical of OA.

\subsection{Summary}

Classically Optic Ataxia has been viewed as not secondary to oculomotor deficits (see Perenin and Vighetto, 1988). However, a recent study (Gaveau et al., 2008) on two optic ataxic patients with bilateral parietal lesions in a natural "look and point" paradigm with a target jump showed a delayed visual capture, which resulted in a delayed pointing to visual targets. Thus, as shown in monkeys, during trajectory corrections, the elongation of the time necessary to reverse an already delayed eye movement largely influences the change of hand movement direction. However, dissociations between impairments in making hand movements and preservation of saccades have also been found in other optic ataxic patients (Khan et al., 2009; Trillenberg et al., 2007).

It remains unclear how central impaired eye movement control is in Optic Ataxia in humans. This can only be answered by means of a sizeable case series, which does not currently exist for this aspect of the syndrome; there is too much reliance on the behavior of a very few single cases. However, even if, as suggested by Gaveau et al. (2008) and Battaglia-Mayer et al. (2007) but rejected by Trillenberg et al. (2007), part of the spatial determination of extent and direction of saccades and rapid manual movements is implemented by the same system, and following Archambault et al. (2009, 2011), this system has the function of trajectory-setting, then the account of Optic Ataxia presented at the end of the previous section can still apply. This is that such a system exists and would need to be controlled by a premotor-located system that becomes critical for prompting the whole parieto-frontal system to be activated when a new motor plan has to be generated or in special circumstances, such as when there is movement of the target.

\section{Explicit suppression of reaching}

\subsection{Neurophysiological studies in animals}

In animal models, most of the studies exploring neural modulations underlying inhibitory control of arm movements have so far used two main paradigms: the Go/No-Go task and the Stop (countermanding) task. Behaviorally, both paradigms give rise to commission errors. However, they explore two different aspects of the explicit suppression of a motor response. In the Go/No-Go paradigms it is a potential and not an ongoing movement that has to be halted. Instead only the Stop task allows one to explore the neural modulation of the timing related to the estimated speed of the presumed stop process, as estimated by use of the so-called Stop Signal Reaction Time (SSRT, Logan and Cowan, 1984; Schall and Godlove, 2012). As discussed above, the Stop task is generally interpreted as involving a simple race model between two independent processes, which are concerned with response execution and inhibition respectively, even if the existence and independence of the two processes has been recently questioned (Boucher et al., 2007; for a related discussion see Bissett 2013). 
It is reasonable to assume that the cortical networks for both action restraining, as in the Go/No-Go task, and action cancelation, as in the Stop task largely overlap. However, most of the available data is focused on the role of a few frontal regions and of their connected subcortical structures, especially those of the corticobasal ganglia loop (Aron and Poldrack, 2006; Li et al., 2006; Schmidt et al., 2013; Vink et al., 2005). The suggested absence of any role for the parietal lobe, stemming from neurophysiological studies using the Go/No-Go task (Kalaska and Crammond, 1995), could be attributed to the fact that in successful trials of both tasks no movement is required and therefore, on the overall theoretical perspective (Scheme 1 ) adopted by this paper, on-line control/update of the movement trajectory is not required. However, it is important to stress that some role of the parietal lobe in both tasks is to be expected (see next section).

In the frontal lobe, M1 represents an important node for all the cortical processes involved in the encoding of arm movement generation. The pyramidal tract (PT) provides both fast-conducting axons originating in M1 and axons with slower conduction velocity originating from other cortical areas (e.g. Kraskov et al., 2009; Maier et al., 2002), and therefore a wide range of conduction delays (Innocenti et al., 2013). However, neurons originating from M1 are more powerful in influencing the spinal cord output not only because of their advantage in terms of conduction delays, but also because they preferentially target spinal cord interneurons (Dum and Strick, 1991). The activity of M1's PT neurons is under the control, presumably, of local interneurons (Reynolds and Ashby, 1999) and a simple gating mechanism requiring that they modify their discharge at the time of movement onset could be postulated. Most of the areas contributing, although with different strength, to the PT also have reciprocal cortico-cortical connections with M1 (Johnson et al., 1996; Johnson and Ferraina, 1996), and by targeting interneurons selectively (Ghosh and Porter, 1988; Tokuno and Nambu, 2000) they can contribute to the gating of movement generation.

Some observations support a controlling role of PMd over M1. The injection of GABA-A antagonists within PMd reduces the ability of monkeys to withhold movements (Sawaguchi et al., 1996). Similarly, lesions to PMd result in an increased frequency of impulsive and uncontrolled reaching movements (Moll and Kuypers, 1977). Pyramidal neurons in PMd could influence inhibitory cells in M1 directly, potentially supporting feed-forward inhibition (Ghosh and Porter, 1988; Tokuno and Nambu, 2000) or they could target inhibitory interneurons at the level of the spinal cord (Dum and Strick, 1991) as indicated in Scheme 1. Recent evidence (Kaufman et al., 2013; Merchant et al., 2008) shows that putative inhibitory interneurons in M1, classified using the trough-to-peak duration of the unfiltered extracellular spike waveform, increase their discharge at the time of movement generation (however, see Vigneswaran et al., 2011 for a specific comments on this method when applied to M1). Different sub-populations of neurons showing preparatory activity during successful inhibition of reaching movements in PMd display either a reduction or an increase in their activity (Mirabella et al., 2011); this is compatible with both of the functional pathways referred to above.

One could assume that when a movement is to be suppressed an increase in the activity of PMd neurons refers to cortico-spinal neurons targeting inhibitory neurons, while PMd neurons that reduce their activity are cortico-cortical neurons targeting M1 interneurons. This would fit with the Boucher et al. (2007) interactive race model at the computational modeling level. In conclusion, where the control gate for reaching arm movements is located, at the cortical or subcortical level, is still a matter of discussion. In fact, a gating effect could be obtained at a very late step of the process by action at the level of the spinal cord, where descending neurons could exert an inhibitory effect on spinal inhibitory circuits (Prut and Fetz, 1999). Similarly, the existence and the role of a gatingbased mechanism for the cortico-cortical influences over the M1's output require further investigation (see also DeLong, 1990).

Most of the studies exploring inhibitory control of arm movement have so far used the Go/No-Go task. Using this task during recording in M1, two different studies (Miller et al., 1992; Port et al., 2001) showed that hand movement per se is not necessary for obtaining activity modulation in M1. In both studies a subpopulation of neurons was found more active during movement suppression than execution. Neurons with similar properties have been reported in premotor cortex (Kalaska and Crammond, 1995; Watanabe, 1986). These latter authors found no such activity in area 5 , suggesting, as discussed above, that it is premotor, not parietal cortex, that is related to No-Go processes (see Scheme 1).

PMd is a well-known target region of prefrontal cortex (PFC). Sasaki and Gemba (1986) recorded No-Go responses using epicortical local field potentials (LFPs) from two prefrontal areas of the monkey's brain, the dorsal bank of the principal sulcus (PS) and the rostro-ventral corner of the prefrontal cortex; their findings suggest that the two areas might play a crucial role in refraining from action. This conclusion was further supported by the use of stimulation procedures in the same areas (Sasaki et al., 1989). A further cortical region under the control of PFC is the most anterior portion of pre-SMA. The presence of neurons discharging during both Go and No-Go trials in pre-SMA (Isoda and Hikosaka, 2007) indicates that this area plays an important role in the control of responses under this task condition.

In summary, findings obtained using the Go/No-Go paradigm support the existence of a frontal network, formed by the PFC, pre-SMA, PMd and M1 concerned with the inhibition of potential responses. In this network a 'No-Go signal' could propagate from more rostral to more caudal regions, as suggested by causality approaches to event-related potentials obtained from epicortical electrodes in monkeys (Zhang et al., 2008). Alternatively, the decision 'not-to-move' could emerge from a late (with a latency of about $150 \mathrm{~ms}$ ) and more parallel organization of a distributed network active at the moment of the decision (Ledberg et al., 2007).

The Stop task is better suited to explore the role of neural populations during the interruption of an already matured motor plan. The modulation of neural activity in both M1 and PMd reflects the local build-up of a motor plan before its execution. It is known that potential motor plans and targets can be simultaneously represented in PMd (Cisek and Kalaska, 2005). However, no studies are yet available in M1 that used the Stop task to investigate inhibitionspecific neural modulation in monkeys.

A recent study (Mirabella et al., 2011) reported a consistent population of neurons in PMd predictive of the animal's success in movement suppression. Modulated neurons had characteristics similar to those of neurons described in the motor areas controlling saccadic generation (Hanes et al., 1998; Paré and Hanes, 2003). Many neurons were modulated after the Stop signal presentation and well before the end of the SSRT. An important difference with the oculomotor neurons is that in PMd (Mirabella et al., 2011) two classes of neurons have been described. In one, reaching-related activity is reduced after Stop signal presentation (as in oculomotor areas; Hanes et al., 1998; Paré and Hanes, 2003), which could fit with the interactive race model referred to above. The second population increases their activity before the end of the estimated time of the Stop process, as one would expect from Stop units. Whether this last class of neurons specifically controls antagonist muscles or represents inhibitory neurons with an indirect control on projection neurons is still an open issue (for a discussion see Mirabella et al., 2011). This argument is closely related to a better understanding of the microcircuit organization of the cerebral cortex. This is starting to be delineated in rodents (e.g. Anderson et al., 2010; Gentet, 2012), but remains to be worked out in detail in primates. 
Neural modulations during motor plan maturation in PMd, studied at the level of multiunit activity, display two stereotyped forms of transition toward either lower or higher states, when compared to the level of activity measured at the time of target presentation (Mattia et al., 2013). These transitions were also observed when the movement was successfully canceled suggesting that they participate causally in that process too, but with a dynamic complementary to that of completing the Go-process.

These results further support the idea that important differences should exist between the cortical control of eye and arm movements. In the frontal eye fields (FEF), different classes of neurons participate in inhibitory control. While visual neurons have no evident role, visuomotor and movement related neurons display a clear contribution, although with some important differences (Ray et al., 2009). Fixation neurons display a reduction of their gazeholding influence during SSRT, as described for fixation neurons in the superior colliculus (Hanes et al., 1998; Paré and Hanes, 2003). In PMd (Mirabella et al., 2011) the existence of two classes of neurons (type A and type B) suggest a further organization. Both types are arm movement-related, but only type A neurons are modulated in the same fashion as the movement neurons in the FEF. Type B neurons represent a class never described in the oculomotor centers.

These results (Mirabella et al., 2011) are also quantitatively different from those reported in SMA and pre-SMA during an arm Stop task. SMA output neurons with preparatory activity target M1 neurons with similar preparatory firing (Aizawa and Tanji, 1994; Tanji and Kurata, 1985). With respect to the Stop task, most of the neural responses in SMA occur after the SSRT (Scangos and Stuphorn, 2010), while a causal relation is observed only in the modulation of local field potentials (LFPs; Chen et al., 2010), which could correspond to an indirect sign (Logothetis, 2003; Mattia et al., 2010) of the Stop-related modulation in remote areas connected to SMA/pre-SMA. A small group of neurons were more active during successful response inhibition, like the fixation neurons in FEF, and a few of them were active early enough to be able to influence the inhibition of the movement.

The modulation observed in the pre-SMA/SMA complex suggests that this region is not directly involved in the control of movement execution. However, the late modulation and the increase of activity in the subpopulation of modulated neurons do indicate a role. Although further studies are necessary, the different pattern of modulation of pre-SMA neurons during the Go/No-Go and Stop tasks suggests that there exists a difference in the distributed network controlling the two forms of motor inhibition studied using the two tasks. One possibility is that SMA activity determines the response threshold for initiating a movement (Chen et al., 2010). A second plausible recent proposal is that a contribution of the pre-SMA/SMA complex is in modulating the proactive control of movement inhibition and participating in performance monitoring (Marcos et al., 2013; Scangos et al., 2013; Stuphorn and Emeric, 2012).

\subsection{Neuropsychological and imaging studies}

Inhibitory control is an important executive function. In a continuously changing environment, updating/selecting representations and goals is critical for the ability to plan and control behavior. Impulsivity in motor acts is found when unmotivated behaviors elicited by the environment alone occur following frontal lesions, particularly of the medial regions, such as in the grasp reflex (De Renzi and Barbieri, 1992) and utilization behavior (Lhermitte, 1983). More generally, perseveration of action schemas is also a well-known consequence of prefrontal lesions (Milner, 1963). Current theories of certain childhood psychopathologies hold deficits to the inhibition structure to be a central aspect (Barkley, 1997;
Quay, 1997), suggesting that inhibitory control develops throughout childhood.

The research literature on the processes involved in the Go/NoGo and Stop tasks have mainly focused on the frontal cortex. As far as the parietal cortex is concerned, imaging studies have shown that the inferior parietal cortex is the region predominantly activated in both tasks (Rubia et al., 2001). We would like to argue that this activation is consistent with a role of this region in the early phases of either Stop signal (or cue signal) identification, in the spatial localization of targets, and in particular in stimulus-driven control of attention (Corbetta and Shulman, 2002; Swick et al., 2011) that the solution of both tasks require. Thus, to be more precise, we conclude that superior parietal regions have no key described role as yet within the parieto-frontal network being discussed.

Returning to the frontal cortex, a prominent hypothesis is that the Stop unit process involved in inhibiting a movement, such as in the Stop signal task, critically involves the right ventrolateral prefrontal cortex in humans. In agreement with this hypothesis, Aron et al. (2003) found that SSRT was 50 ms longer for right (but not left) frontal patients than controls. In addition the highest correlation between size of lesion in a region and SSRT was for the right inferior frontal gyrus $(r=0.83)$, although the correlation was significant for the right middle frontal gyrus too. This finding was not, however, corroborated in a study of 23 frontal patients by Floden and Stuss (2006). Of the 7 patients with SSRTs more than 1.5 the control mean, 5 had lesions to the superior medial region with maximal overlap in the pre-SMA and SMA. A more recent neuropsychological investigation of the task in head injury patients concerned the involvement of five white matter tracts (Bonnelle et al., 2012). Again only the right anterior insula to pre-SMA/anterior cingulate tract showed a significant relation with SSRT. Two tracts involving the right inferior frontal gyrus did not.

Similarly, two TMS studies have focused on determining the relative roles of right ventrolateral region or more medial regions in the Stop signal task. The study of Chambers et al. (2006) led to unclear results. In block 1 , of 128 trials, when single pulse TMS was administered to the right inferior frontal gyrus, they found an increased SSRT, when compared to TMS to control regions and sham. However, the effect disappeared in block 2. Rather analogous findings were obtained by Chen et al. (2009) with TMS to the pre-SMA. One explanation is that processes other than response inhibition come into play for the first block only, presumably to do with the novel learning situation. Alternatively functional reorganization might have taken place and then the first block findings are more critical.

In a further study which used rTMS carried out before the blocks of trials, Chambers et al. (2007) employed a more complex basic task - the Eriksen flanker task. Two experiments were carried out, one in each hemisphere. For right ventrolateral stimuli but not left, there was a significant interaction between locus of TMS and whether flanking stimuli were congruent or incongruent; this locus produced a small $15 \mathrm{~ms}$ slowing compared with the other two loci when inappropriate response tendencies must be inhibited. Thus the TMS studies do not provide strong support for a right ventrolateral prefrontal localization of a Stop unit process.

Mattia et al. (2012) recorded ERPs from the lateral surface of the frontal cortex in five epileptic patients. A steoreotyped ERP complex was found when successful countermanding occurred in the Stop task. The use of subdural grids for recording allowed higher spatial resolution than normal with EEG; the areas involved in the Stop complex were M1, premotor cortex and area 9, but not the ventrolateral PFC.

On the ventrolateral Stop unit hypothesis, it remains unclear how the inhibitory function might be implemented. Aron and Poldrack (2006) argued that the subthalamic nucleus (STN) might play a role as it could suppress the effect of the "direct" 
fronto-striatal pathway activated by response initiation. Using fMRI they found that there was also activation of STN in the Stop condition. However, this STN effect was not replicated by Hampshire et al. (2010).

More neuropsychological studies have been carried out on the other key paradigm - Go/No-Go. Early studies (e.g. Decary and Richer, 1995; Drewe, 1975) showed that frontal patients were more impaired on the Go/No-Go task than patients with nonfrontal lesions. Some early studies (Godefroy and Rousseaux, 1996; Leimkuhler and Mesulam, 1985; Verfaellie and Heilman, 1987) also provided some indication of localization within frontal cortex, but with small numbers of patients. All three pointed to a specifically medial frontal involvement rather than a lateral frontal one. The stress on the relevance of medial frontal regions was supported by much the largest frontal lobe study ( 43 frontal patients) of the Go/No-Go task, that of Picton et al. (2007). They found that when the right hand was used, commission errors were significantly greater in patients with left superior medial frontal lesions, affecting areas $6 a$ and 8 (including the SMA and the pre-SMA) or with left dorsal premotor lesions than in patients with lesions to other parts of the frontal lobes. By contrast, there was no effect of lesions to the right ventrolateral region on commission errors.

Many functional imaging studies have been carried out on the Go/No-Go and Stop signal tasks. Thus one review (Levy and Wagner, 2011) lists 32 Go/No-Go and 17 Stop signal studies. However, three recent meta-analyses (Criaud and Boulinguez, 2013; Levy and Wagner, 2011; Swick et al., 2011) come to quite different conclusions regarding the localization of any Stop unit. The Levy-Wagner meta-analysis shows that many frontal regions are significantly activated including the right posterior and mid-ventrolateral prefrontal cortex, the right anterior cingulate, both pre-SMAs and both dorsal premotor cortices. It is this variety of loci, which makes interpretation of the functions involved in the different regions difficult.

The role of the right ventrolateral cortex in Stop signal and Go/No-Go studies has been particularly controversial. Two main perspectives have been put forward. One, that of Levy and Wagner, is that different sub regions of the ventrolateral prefrontal cortex are involved, one for the inhibiting of motor responses and the second for bottom-up attentional capture. The more popular view is that the inferior frontal gyrus is, however, specifically involved in attentional capture but not in inhibitory control, as indicated in Scheme 1. Thus Sharp et al. (2010) used a basic task where subjects must respond to left and right pointing arrows for the on-line task with a circle occurring on some trials (Fig. 5). If the circle was red it was to be treated as a Stop signal, but if green the response was to proceed as normal (continue). The right inferior prefrontal gyrus (RIFG) was activated in both these conditions. Regions activated more for Stop included pre-SMA and lateral SMA, but not the RIFG. Similar conclusions can be drawn from the studies of Dodds et al. (2011), Hampshire et al. (2010), and Verbruggen et al. (2010). Probably more relevant for neurophysiological studies of the Stop signal paradigm, Chao et al. (2009) found that the pre-SMA, but not the RIFG, showed greater activity in individuals with short as compared to those with long SSRT (see also Swick et al., 2011). Thus we will assume that any Stop unit is not located in ventrolateral prefrontal cortex.

In their metaanalysis, Criaud and Boulinguez (2013), find that the pre-SMA is also activated more for complex rather than simple Go/No-Go designs and for low as opposed to high frequency No-Go signals. In part these effects may be a consequence of cascading inputs from higher-level systems. However, the authors argue that the pre-SMA "contributes to many reactive functions that are involved in cognitive control" (p. 19), including adjusting response thresholds. This is somewhat similar to the ideas of Scangos and colleagues from the neurophysiological literature on the pre-SMA/SMA complex.

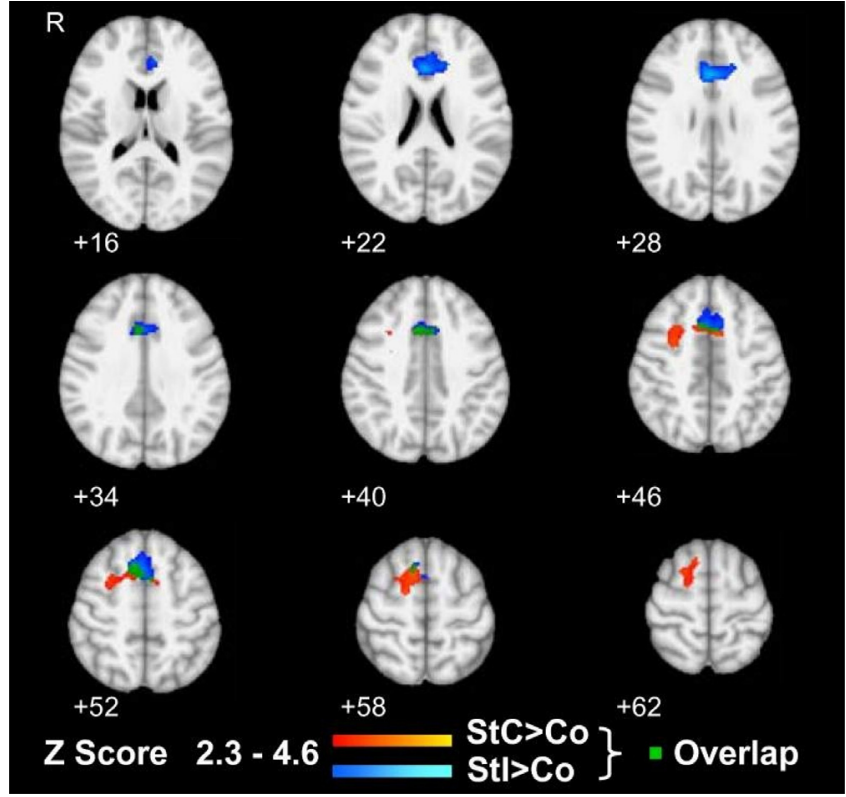

Fig. 5. Nine axial slices showing areas with significantly higher activation on Stop trials than Continue trials in the functional imaging study of Sharp et al. (2010). The red areas, including the right medial and lateral pre-SMA and the right lateral SMA, are regions that are more activated for successful Stop responses than Continue responses. The blue areas, principally in the anterior cingulate cortex, are regions more activated for unsuccessful Stop responses than Continue responses. For green areas both of the above contrasts held. Note there is no activation of the right inferior frontal gyrus. The images follow radiological convention in being left-right reversed. Source: Reproduced from Sharp et al. (2010).

\subsection{Summary}

In this section we considered the structures involved in two different action suppression tasks - Go/No-Go and the Stop signal tasks. Models of performance in these tasks involve the hypothesis of a critical Stop unit system. The structures most investigated in monkey with respect to the possible location of a Stop unit system are motor cortex, dorsal premotor cortex and pre-SMA. In particular, one set of neurons in the dorsal premotor cortex behave as one would expect from the operation of a Stop unit. The possible roles in these tasks of the pre-SMA/SMA complex are less clear with different possibilities having been put forward. As discussed just above, in humans the latter two regions are indeed found to result, when lesioned, in commission errors (for the Go/No-Go task) and also to be activated in functional imaging (for both tasks). Moreover a region little investigated in the monkey in this context, the ventrolateral prefrontal cortex, has been held to be critical for response inhibition in the Stop task for humans, but more recently it has been more frequent to view the involvement of this region in this task as due to bottom-up attentional capture. Overall, the human and monkey evidence on explicit suppression lead to similar conclusions.

\section{Conclusions}

The literature reviewed in this paper provides converging evidence across a range of disciplines and procedures on the basic mechanisms involved in correcting a reach when it has to be changed in direction or inhibited with particular reference to the roles of the ventrolateral prefrontal, premotor, motor, and parietal cortices. Moreover, despite the apparent differences that exist between the monkey and the human in the organization of premotor and particularly the parietal cortex, it now appears that there is a basic similarity, not identity, in the anatomies of these 
two critical regions across these species (Caspers et al., 2011; Sallet et al., 2013). Thus one can draw inferences across the two about the basic organization and mechanisms involved in the control of reaching. In addition, the three tasks considered in detail in this paper - the Double-Step task, the Go/No-Go task and the Stop signal task - appear to involve a basically similar set of mechanisms.

From behavioral investigations, it has been proposed that the limb movements involved are based on a small set of time-varying activation patterns, each involving multiple muscles. Moreover, at this level, it appears that on-line correction of reaching uses the same set of muscle synergies as uncorrected reaching, appropriately modulated in amplitude and timing. This has the corollary that higher-level control of the modulation of reaching is likely to be different if reaching is instead mimicked for technical reasons by some non-standard means, such as by the use of a joystick. We thus confine consideration only to studies using a standard reaching procedure.

The key structures as far as the modulation of the reaching movement are concerned are the superior parietal and dorsal premotor cortices. If a target moves to a new position when the reach is being triggered, both the potential trajectories are represented in the parietal and premotor cortices. From neurophysiological evidence, the trajectory of an uncorrected reach appears to be initially specified in the superior parietal cortex, and the neuropsychological evidence is compatible with this position. Moreover, both the trajectories of the smooth corrected action and the activity of superior parietal neurons can be predicted from what is known of the neurophysiological response to an action when the target does not move. Thus as far as the parietal cortex is concerned, the neurophysiological evidence suggests that there is no special system that comes into play when movements have to be corrected or inhibited.

What systems then are responsible for the change in behavior that the critical stimuli induce? Our overall perspective is illustrated in Scheme 1. In all three tasks, it is clear that the dorsal premotor cortex plays a critical role in the initiation or suppression of a new or altered motor plan. This can be in a positive fashion by its requiring the parietal cortex to specify a new trajectory in the Double-Step task. Alternatively in negative fashion the very same area of dorsal premotor cortex triggers inhibitory processes in motor cortex and the spinal cord in the Go/No-Go and Stop tasks. Moreover, it appears that related biased competition and interactive race models can be employed to account for dorsal premotor functioning both in the correction of reaching and in explicit suppression. Differences do occur between the three tasks as far as systems upstream of premotor cortex are concerned. For the Go/No-Go task, the pre-SMA also seems to be critical, but from the neurophysiological evidence this is less clear for the Stop signal task.

A more concrete proposal of Mattia et al. (2013) is that the PMd control processes are mediated by attractor-based systems. Individual attractors could then represent both the motor plan and any spatially specified target, the former as a point attractor and the latter as a plane attractor. In this case additional systems, such as the pre-SMA for the Go/No-Go task, could also be involved in the neural network forming the anatomical bases of the attractor for any individual task. If the target is represented through a plane attractor then changes of continuous or discontinuous form can be implemented using essentially the same system.

In recent years there have been influential suggestions that this essentially simple scheme needs to be made more complex. Thus from a variety of methodologies with human subjects, it has been argued that there are systems in parietal cortex that come into play when reaches must be corrected. However, the relevant functional imaging and TMS studies use non-standard reaching tasks and the neuropsychological single case evidence is not corroborated by patient group findings. Similarly, it has been argued from neuropsychological and imaging studies in humans that the right ventrolateral prefrontal cortex contains a Stop unit type of system. This evidence has since been interpreted in other ways; the majority view now is that this region has an attentional function rather than an inhibitory control one.

We therefore have converging evidence across both tasks and methodologies of the roles of different brain regions in the on-line correction and inhibition of reaching. Eventual and countermanded actions use the same set of muscle synergies and appear to be represented in parallel in the key cortical structures. Thus, the initial specification of the trajectories required in setting up a reach and in correcting it appears to be carried out solely in the superior parietal cortex. Then there is a central role for systems primarily based in the same region of dorsal premotor cortex in initiating both motor plan correction and in suppression.

\section{Acknowledgments}

This work was partially supported by the MIUR of Italy (prot. 2010MEFNF7_004 to RC, and prot. 2008J7YFNR_004 to TS), by the Italian Space Agency (DCMC and CRUSOE grants to FL), and by the European Commission (FP7 Collaborative Research Project BRAINLEAP - ref. 306502 - to SF). We are grateful to Prof. Y. Rossetti for providing the material used in Fig. 4.

\section{References}

Aizawa, H., Tanji, J., 1994. Corticocortical and thalamocortical responses of neurons in the monkey primary motor cortex and their relation to a trained motor task. J. Neurophysiol. 71, 550-560.

Anderson, C.T., Sheets, P.L., Kiritani, T., Shepherd, G.M., 2010. Sublayer-specific microcircuits of corticospinal and corticostriatal neurons in motor cortex. Nat. Neurosci. 13, 739-744.

Archambault, P.S., Caminiti, R., Battaglia-Mayer, A., 2009. Cortical mechanisms for on-line control of hand movement trajectory: the role of the posterior parietal cortex. Cereb. Cortex 19, 2848-2864.

Archambault, P.S., Ferrari-Toniolo, S., Battaglia-Mayer, A., 2011. On-line control of hand trajectory and evolution of motor intention in the parietofrontal system. J. Neurosci. 31, 742-752.

Aron, A.R., Fletcher, P.C., Bullmore, E.T., Sahakian, B.J., Robbins, T.W., 2003. Stopsignal inhibition disrupted by damage to right inferior frontal gyrus in humans Nat. Neurosci. 6, 115-116.

Aron, A.R., Poldrack, R.A., 2006. Cortical and subcortical contributions to stop signal response inhibition: role of the subthalamic nucleus. J. Neurosci. 26, 2424-2433.

Averbeck, B.B., Battaglia-Mayer, A., Guglielmo, C., Caminiti, R., 2009. Statistical analysis of the parieto-frontal cognitive-motor network. J. Neurophysiol. 102 1911-1920

Bard, C., Turrell, Y., Fleury, M., Teasdale, N., Lamarre, Y., Martin, O., 1999. Deafferentation and pointing with visual double-step perturbations. Exp. Brain Res. 125 410-416.

Barkley, R.A., 1997. ADHD and the Nature of Self-control. Guilford, New York.

Battaglia-Mayer, A., Caminiti, R., Lacquaniti, F., Zago, M., 2003. Multiple levels of representation of reaching in the parieto-frontal network. Cereb. Cortex 13, 1009-1022.

Battaglia-Mayer, A., Mascaro, M., Caminiti, R., 2007. Temporal evolution and strength of neural activity in parietal cortex during eye and hand movements. Cereb. Cortex 17, 1350-1363.

Battaglia-Mayer, A., Ferrari-Toniolo, S., Visco-Comandini, F., Archambault, P.S. Saberi-Moghadam, S., Caminiti, R., 2012. Impairment of on-line control of hand and eye movements in a monkey model of optic ataxia. Cereb. Cortex 23 2644-2656.

Beggs, W.D., Howarth, C.I., 1972. The accuracy of aiming at a target. Some furthe evidence for a theory of intermittent control. Acta Psychol. 36, 171-177.

Berry, M.J., Brivanlou, I.H., Jordan, T.A., Meister, M., 1999. Anticipation of moving stimuli by the retina. Nature $398,334-338$.

Blangero, A., Gaveau, V., Luauté, J., Rode, G., Salemme, R., Guinard, M., Boisson, D. Rossetti, Y., Pisella, L., 2008. A hand and a field effect in on-line motor control in unilateral optic ataxia. Cortex 44, 560-568.

Blangero, A., Menz, M.M., McNamara, A., Binkofski, F., 2009. Parietal modules for reaching. Neuropsychologia 47, 1500-1507.

Bonnelle, V., Ham, T.E., Leech, R., Kinnunen, K.M., Mehta, M.A., Greenwood, R.J., Sharp, D.J., 2012. Salience network integrity predicts default mode network function after traumatic brain injury. Proc. Natl. Acad. Sci. U.S.A. 109, 4690-4695

Boucher, L., Palmeri, T.J., Logan, G.D., Schall, J.D., 2007. Inhibitory control in mind and brain: an interactive race model of countermanding saccades. Psychol. Rev. 114, 376-397. 
Brenner, E., Smeets, J.B., 1997. Fast responses of the human hand to changes in target position. J. Mot. Behav. 29, 297-310.

Buiatti, T., Skrap, M., Shallice, T., 2013. Reaching a moveable visual target: dissociations in brain tumour patients. Brain Cogn. 82, 6-17.

Bullock, D., Grossberg, S., 1988. Neural dynamics of planned arm movements: emergent invariants and speed-accuracy properties during trajectory formation. Psychol. Rev. 95, 49-90.

Busan, P., Barbera, C., Semenic, M., Monti, F., Pizzolato, G., Pelamatti, G., Battaglini, P.P., 2009. Effect of transcranial magnetic stimulation (TMS) on parietal and premotor cortex during planning of reaching movements. PLoS ONE 4, e4621.

Buxbaum, L.J., Coslett, H.B., 1998. Spatio-motor representations in reading: evidence for subtypes of optic ataxia. Cogn. Neuropsychol. 15, 279-312.

Camalier, C.R., Gotler, A., Murthy, A., Thompson, K.G., Logan, G.D., Palmeri, T.J., Schall, J.D., 2007. Dynamics of saccade target selection: race model analysis of double step and search step saccade production in human and macaque. Vision Res. 47, 2187-2211.

Cameron, B.D., Cressman, E.K., Franks, I.M., Chua, R., 2009. Cognitive constraint on the 'automatic pilot' for the hand: movement intention influences the hand's susceptibility to involuntary on-line corrections. Conscious Cogn. 18, 646-652.

Caminiti, R., Ferraina, S., Johnson, P.B., 1996. The sources of visual information to the primate frontal lobe: a novel role for the superior parietal lobule. Cereb. Cortex 6, 319-328.

Caminiti, R., Ghaziri, H., Galuske, R., Hof, P.R., Innocenti, G.M., 2009. Evolution amplified processing with temporally-dispersed, slow, neural connectivity in primates. Proc. Natl. Acad. Sci. U.S.A. 106, 19551-19556.

Caspers, S., Eickhoff, S.B., Rick, T., von Kapri, A., Kuhlen, T., Huang, R., Shah, N.J., Zilles, K., 2011. Probabilistic fibre tract analysis of cytoarchitectonically defined human inferior parietal lobule areas reveals similarities to macaques. Neuroimage 58 , 362-380.

Chambers, C.D., Bellgrove, M.A., Gould, I.C., English, T., Garavan, H., McNaught, E., Kamke, M., Mattingley, J.B., 2007. Dissociable mechanisms of cognitive control in prefrontal and premotor cortex. J. Neurophysiol. 98, 3638-3647.

Chambers, C.D., Bellgrove, M.A., Stokes, M.G., Henderson, T.R., Garavan, H., Robertson, I.H., Morris, A.P., Mattingley, J.B., 2006. Executive brake failure following deactivation of human frontal lobe. J. Cogn. Neurosci. 18, 444-455.

Chao, H.H., Luo, X., Chang, J.L., Li, C.S., 2009. Activation of the pre-supplementary motor area but not inferior prefrontal cortex in association with short stop signal reaction time-an intra-subject analysis. BMC Neurosci. 10, 75.

Chen, C.Y., Muggleton, N.G., Tzeng, O.J.L., Hung, D.L., Juan, C.H., 2009. Control of prepotent responses by the superior medial frontal cortex. Neuroimage 44, 537-545.

Chen, X., Scangos, K.W., Stuphorn, V., 2010. Supplementary motor area exerts proactive and reactive control of arm movements. J. Neurosci. 30, 1465714675.

Cisek, P., Kalaska, J.F., 2005. Neural correlates of reaching decisions in dorsal premotor cortex: specification of multiple direction choices and final selection of action. Neuron 45, 801-814.

Clower, D.M., Hoffman, J.M., Votaw, J.R., Faber, T.L., Woods, R.P., Alexander, G., 1996. Role of posterior parietal cortex in the recalibration of visually guided reaching. Nature 383, 618-621.

Conti, F., Fabri, M., Manzoni, T., 1988. Immunocytochemical evidence for glutamatergic cortico-cortical connections in monkey. Brain Res. 462, 148-153.

Corbetta, M., Shulman, G., 2002. Control of goal-directed and stimulus-driven attention in the brain. Nat. Rev. Neurosci. 3, 201-215.

Coulthard, E., Parton, A., Husain, M., 2006. Action control in visual neglect. Neuropsychologia 44, 2717-2733.

Coxon, J.P., Stinear, C.M., Byblow, W.D., 2006. Intracortical inhibition during volitional inhibition of prepared action. J. Neurophysiol. 95, 3371-3383.

Crawford, J.D., Henriques, D.Y., Medendorp, W.P., 2011. Three-dimensional transformations for goal-directed action. Annu. Rev. Neurosci. 34, 309-331.

Criaud, M., Boulinguez, P., 2013. Have we been asking the right questions when assessing response inhibition in go/no-go tasks with fMRI? A meta-analysis and critical review. Neurosci. Biobehav. Rev. 37, 11-23.

d'Avella, A., Fernandez, L., Portone, A., Lacquaniti, F., 2008. Modulation of phasic and tonic muscle synergies with reaching direction and speed. J. Neurophysiol. 100, 1433-1454.

d'Avella, A., Lacquaniti, F., 2013. Control of reaching movements by muscle synergy combinations. Front. Comput. Neurosci. 7, 42.

d'Avella, A., Portone, A., Fernandez, L., Lacquaniti, F., 2006. Control of fast-reaching movements by muscle synergy combinations. J. Neurosci. 26, 7791-7810.

d'Avella, A., Portone, A., Lacquaniti, F., 2011. Superposition and modulation of muscle synergies for reaching in response to a change in target location. J. Neurophysiol. $106,2796-2812$.

Day, B.L., Lyon, I.N., 2000. Voluntary modification of automatic arm movements evoked by motion of a visual target. Exp. Brain Res. 130, 159-168.

Decary, A., Richer, F., 1995. Response selection deficits in frontal excisions. Neuropsychologia 33, 1243-1253.

Della Maggiore, V., Malfait, N., Ostry, D.J., Paus, T., 2004. Stimulation of the posterior parietal cortex interferes with arm trajectory adjustments during the learning of new dynamics. J. Neurosci. 24, 9971-9976.

DeLong, M.R., 1990. Primate models of movement disorders of basal ganglia origin. Trends Neurosci. 13, 281-285.

De Renzi, E., Barbieri, C., 1992. The incidence of the grasp reflex following hemispheric lesion and its relation to frontal damage. Brain 115, 293-313.
Desmurget, M., Epstein, C.M., Turner, R.S., Prablanc, C., Alexander, G.E., Grafton, S.T . 1999. Role of the posterior parietal cortex in updating reaching movements to a visual target. Nat. Neurosci. 2, 563-567.

Desmurget, M., Grafton, S., 2000. Forward modeling allows feedback control for fast reaching movements. Trends Cogn. Sci. 4, 423-431.

Desmurget, M., Gréa, H., Grethe, J.S., Prablanc, C., Alexander, G.E., Grafton, S.T., 2001 Functional anatomy of nonvisual feedback loops during reaching: a positron emission tomography study. J. Neurosci. 21, 2919-2928.

Dickey, A.S., Amit, Y., Hatsopoulos, N.G., 2013. Heterogeneous neural coding of corrective movements in motor cortex. Front. Neural Circ. 7, 51.

Dimitriou, M., Wolpert, D.M., Franklin, D.W., 2013. The temporal evolution of feedback gains rapidly update to task demands. J. Neurosci. 33, 10898-10909.

Dodds, C.M., Morein-Zamir, S., Robbins, T.W., 2011. Dissociating inhibition, attention, and response control in the frontoparietal network using functional magnetic resonance imaging. Cereb. Cortex 21, 1155-1165.

Drewe, E.A., 1975. Go-no go learning after frontal lobe lesions in humans. Cortex 11 $8-16$.

Dum, R.P., Strick, P.L., 1991. The origin of corticospinal projections from the premotor areas in the frontal lobe. J. Neurosci. 11, 667-689.

Ferraina, S., Brunamonti, E., Giusti, M.A., Costa, S., Genovesio, A., Caminiti, R., 2009. Reaching in depth: hand position dominates over binocular eye position in the rostral superior parietal lobule. J. Neurosci. 29, 11461-11470.

Fautrelle, L., Ballay, Y., Bonnetblanc, F., 2010. Muscular synergies during motor corrections: investigation of the latencies of muscle activities. Behav. Brain Res. $214,428-436$

Flash, T., Henis, E., 1991. Arm trajectory modifications during reaching towards visual targets. J. Cogn. Neurosci. 3, 220-230.

Floden, D., Stuss, D.T., 2006. Inhibitory control is slowed in patients with right superior medial frontal damage. J. Cogn. Neurosci. 18, 1843-1849.

Franklin, D.W., Wolpert, D.M., 2011. Computational mechanisms of sensorimotor control. Neuron 72, 425-442.

Gaveau, V., Pélisson, D., Blanghero, A., Urquizar, C., Prablanc, C., Vighetto, A., Pisella, L., 2008. Saccade control and eye-hand coordination in optic ataxia. Neuropsychologia 46, 475-486.

Gentet, L.J., 2012. Functional diversity of supragranular GABAergic neurons in the barrel cortex. Front. Neural Circ. 6, 52.

Georgopoulos, A.P., Kalaska, J.F., Caminiti, R., Massey, J.T., 1983. Interruption of motor cortical discharge subserving aimed arm movements. Exp. Brain Res. 49, 327-340.

Georgopoulos, A.P., Kalaska, J.F., Massey, J.T., 1981. Spatial trajectories and reaction times of aimed movements: effects of practice, uncertainty, and change in target location. J. Neurophysiol. 46, 725-743.

Ghosh, S., Porter, R., 1988. Corticocortical synaptic influences on morphologically identified pyramidal neurones in the motor cortex of the monkey. J. Physiol 400, 617-629.

Gielen, C.C., van den Heuvel, P.J., Denier van der Gon, J.J., 1984. Modification of muscle activation patterns during fast goal-directed arm movements. J. Mot. Behav. 16 2-19.

Godefroy, O., Rousseaux, M., 1996. Divided and focused attention in patients with lesion of the prefrontal cortex. Brain Cogn. 30, 155-174.

Gold, J.I., Shadlen, M.N., 2007. The neural basis of decision making. Annu. Rev. Neurosci. 30, 535-574.

Gomi, H., 2008. Implicit online corrections of reaching movements. Curr. Opin. Neurobiol. $18,558-564$.

Goodale, M.A., Pelisson, D., Prablanc, C., 1986. Large adjustments in visually guided reaching do not depend on vision of the hand or perception of target displacement. Nature 320, 748-750.

Gréa, H., Pisella, L., Rossetti, Y., Desmurget, M., Tilikete, C., Grafton, S., Prablanc C., Vighetto, A., 2002. A lesion of the posterior parietal cortex disrupts on-line adjustments during aiming movements. Neuropsychologia 40, 2471-2480.

Gritsenko, V., Yakovenko, S., Kalaska, J.F., 2009. Integration of predictive feedforward and sensory feedback signals for online control of visually guided movement. J. Neurophysiol. 102, 914-930.

Hampshire, A., Chamberlain, S.R., Monti, M.M., Duncan, J., Owen, A.M., 2010. The role of the right inferior frontal gyrus: inhibition and attentional control. Neuroimage 50, 1313-1319.

Hanes, D.P., Patterson 2nd, W.F., Schall, J.D., 1998. Role of frontal eye fields in countermanding saccades: visual, movement, and fixation activity. J. Neurophysiol. 79, 817-834.

Heekeren, H.R., Marrett, S., Ungerleider, L.G., 2011. The neural systems that mediate human perceptual decision making. Nat. Rev. Neurosci. 9, 467-479.

Henis, E.A., Flash, T., 1995. Mechanisms underlying the generation of averaged modified trajectories. Biol. Cybern. 72, 407-419.

Herman, R., Herman, R., Maulucci, R., 1981. Visually triggered eye-arm movements in man. Exp. Brain Res. 42, 392-398.

Hoff, B., Arbib, M.A., 1993. Models of trajectory formation and temporal interaction of reach and grasp. J. Mot. Behav. 25, 175-192.

Hwang, E.H., Hauschild, M., Wilke Andersen, R.A., 2012. Inactivation of the Parietal Reach Region causes optic ataxia, impairing reaches but not saccades. Neuron $76,1021-1029$

Innocenti, G.M., Vercelli, A., Caminiti, R., 2013. The diameter of cortical axons depends both on area of origin and target. Cereb. Cortex http://dx.doi.org/10.1093/cercor/bht070.

Isoda, M., Hikosaka, O., 2007. Switching from automatic to controlled action by monkey medial frontal cortex. Nat. Neurosci. 10, 240-248. 
Izawa, J., Shadmehr, R., 2008. On-line processing of uncertain information in visuomotor control. J. Neurosci. 28, 11360-11368.

Johnson, P.B., Ferraina, S., 1996. Cortical networks for visual reaching: intrinsic frontal lobe connectivity. Eur. J. Neurosci. 8, 1358-1362.

Johnson, P.B., Ferraina, S., Bianchi, L., Caminiti, R., 1996. Cortical networks for visual reaching. Physiological and anatomical organization of frontal and parietal lobe arm regions. Cereb. Cortex 6, 102-119.

Kalaska, J.F., Crammond, D.J., 1995. Deciding not to go: neuronal correlates of response selection in a go/no-go task in primate premotor and parietal cortex. Cereb. Cortex 5, 410-428.

Kane, A., Wade, A., Ma-Wyatt, A., 2011. Delays in using chromatic and luminance information to correct rapid reaches. J. Vis. 11 (September (10)).

Karnath, H.O., Perenin, M.T., 2005. Cortical control of visually guided reaching: evidence from patients with optic ataxia. Cereb. Cortex 15, 15611569.

Kaufman, M.T., Churchland, M.M., Shenoy, K.V., 2013. The roles of monkey M1 neuron classes in movement preparation and execution. J. Neurophysiol. 110, 817-825.

Kawato, M., 1999. Internal models for motor control and trajectory planning. Curr. Opin. Neurobiol. 9, 718-727.

Khan, A.Z., Blangero, A., Rossetti, Y., Salemme, R., Luarte, J., Deubel, H., Schneider, W.X., Laverdure, N., Rode, G., Boisson, D., Pisella, L., 2009. Parietal damage dissociates saccade planning from presacccadic perceptual facilitation. Cereb. Cortex $19,383-387$

Körding, K.P., Wolpert, D.M., 2004. Bayesian integration in sensorimotor learning. Nature 427, 244-247.

Kraskov, A., Dancause, N., Quallo, M.M., Shepherd, S., Lemon, R.N., 2009. Corticospinal neurons in macaque ventral premotor cortex with mirror properties: a potential mechanism for action suppression? Neuron 64, 922-930.

Lacquaniti, F., 1997. Frames of reference in sensorimotor coordination. In: Boller, F., Grafman, J. (Eds.), Handbook of Neuropsychology. Elsevier, Amsterdam (the Netherlands), pp. 27-64.

Lamme, V.A., Roelfsema, P.R., 2000. The distinct modes of vision offered by feedforward and recurrent processing. Trends Neurosci. 23, 571-579.

Ledberg, A., Bressler, S.L., Ding, M., Coppola, R., Nakamura, R., 2007. Largescale visuomotor integration in the cerebral cortex. Cereb. Cortex 17, 44-62.

Lee, J.H., van Donkelaar, P., 2006. The human dorsal premotor cortex generates on-line error corrections during sensorimotor adaptation. J. Neurosci. 26, 3330-3334.

Leimkuhler, M.E., Mesulam, M.M., 1985. Reversible Go-No Go deficits in a case of frontal lobe tumor. Ann. Neurol. 18, 617-619.

Lemon, R.N., 2008. Descending pathways in motor control. Annu. Rev. Neurosci. 31, $195-218$.

Levy, B.J., Wagner, A.D., 2011. Cognitive control and right ventrolateral prefrontal cortex: reflexive reorienting, motor inhibition, and action updating. Ann. N.Y. Acad. Sci. 1224, 40-62.

Lhermitte, F., 1983. Utilization behavior and its relation to lesions of the frontal lobes. Brain 106, 237-255.

Li, C.S., Huang, C., Constable, R.T., Sinha, R., 2006. Imaging response inhibition in a stop-signal task: neural correlates independent of signal monitoring and postresponse processing. J. Neurosci. 26, 186-192.

Liu, D., Todorov, E., 2007. Evidence for the flexible sensorimotor strategies predicted by optimal feedback control. J. Neurosci. 27, 9354-9368.

Livingstone, M., Hubel, D., 1988. Segregation of form, color, movement, and depth: anatomy, physiology, and perception. Science 240, 740-749.

Logan, G.D., Cowan, W.B., 1984. On the ability to inhibit thought and action: a theory of an act of control. Psychol. Rev. 91, 295-327.

Logothetis, N.K., 2003. The underpinnings of the BOLD functional magnetic resonance imaging signal. J. Neurosci. 23, 3963-3971.

Loram, I.D., Gollee, H., Lakie, M., Gawthrop, P.J., 2011. Human control of an inverted pendulum: is continuous control necessary? Is intermittent control effective? Is intermittent control physiological? J. Physiol. 589, 307-324.

Loram, I.D., Lakie, M., Gawthrop, P.J., 2009. Visual control of stable and unstable loads: what is the feedback delay and extent of linear time-invariant control? J. Physiol. 587, 1343-1365.

Maier, M.A., Armand, J., Kirkwood, P.A., Yang, H.W., Davis, J.N., Lemon, R.N., 2002. Differences in the corticospinal projection from primary motor cortex and supplementary motor area to macaque upper limb motoneurons: an anatomical and electrophysiological study. Cereb. Cortex 12, 281-296.

Marconi, B., Genovesio, A., Battaglia-Mayer, A., Ferraina, S., Squatrito, S., Molinari, M., Lacquaniti, F., Caminiti, R., 2001. Eye-hand coordination during reaching. I. Anatomical relationships between parietal and frontal cortex. Cereb. Cortex 11, 513-527.

Marcos, E., Pani, P., Brunamonti, E., Deco, G., Ferraina, S., Verschure, P., 2013. Neural variability in premotor cortex is modulated by trial history and predicts behavioral performance. Neuron 78, 249-255.

Martin, J.A., Himmelbach, M., 2011. Functional representations for reaching to visual targets in central and peripheral vision. Poster Presented at the 3rd Scientific Meeting of the ESN, Federation of European Societies of Neuropsychology, Basel (Ch).

Mattia, M., Ferraina, S., Del Giudice, P., 2010. Dissociated multi-unit activity and local field potentials: a theory inspired analysis of a motor decision task. Neuroimage $52,812-823$.

Mattia, M., Spadacenta, S., Pavone, L., Quarato, P., Esposito, V., Sparano, A., Sebastiano, F., Di Gennaro, G., Morace, R., Cantore, G., Mirabella, G., 2012. Stop-event-related potentials from intracranial electrodes reveal a key role of premotor and motor cortices in stopping ongoing movements. Front Neuroeng. 5, 12.

Mattia, M., Pani, P., Mirabella, G., Costa, S., Del Giudice, P., Ferraina, S., 2013. Heterogeneous attractor cell assemblies for motor planning in premotor cortex. J. Neurosci. 33, 11155-11168.

Maunsell, J.H., Nealey, T.A., DePriest, D.D., 1990. Magnocellular and parvocellular contributions to responses in the middle temporal visual area (MT) of the macaque monkey. J. Neurosci. 10, 3323-3334

McGuire, L.M., Sabes, P.N., 2009. Sensory transformations and the use of multiple reference frames for reach planning. Nat. Neurosci. 12, 1056-1061.

McIntosh, R.D., Mulroue, A., Blangero, A., Pisella, L., Rossetti, Y., 2011. Correlated deficits of perception and action in optic ataxia. Neuropsychologia 49, 131-137.

McIntosh, R.D., Mulroue, A., Brockmole, J.R., 2010. How automatic is the hand's automatic pilot? Evidence from dual-task studies. Exp. Brain Res. 206, 257-269.

Megaw, E.D., 1974. Possible modification to a rapid on-going programmed manual response. Brain Res. 71, 425-441.

Merchant, H., Naselaris, T., Georgopoulos, A.P., 2008. Dynamic sculpting of directional tuning in the primate motor cortex during three-dimensional reaching. J. Neurosci. 28, 9164-9172.

Merigan, W.H., Maunsell, J.H., 1993. How parallel are the primate visual pathways? Annu. Rev. Neurosci. 16, 369-402.

Miller, J., Riehle, A., Requin, J., 1992. Effects of preliminary perceptual output on neuronal activity of the primary motor cortex. J. Exp. Psychol. Hum. 18, 1121-1138.

Milner, B., 1963. Effects of different brain lesions on card sorting. The role of fronta lobes. Arch. Neurol. 9, 90-100.

Mirabella, G., Pani, P., Ferraina, S., 2011. Neural correlates of cognitive control of reaching movements in the dorsal premotor cortex of rhesus monkeys. J. Neurophysiol. 106, 1454-1466.

Moll, L., Kuypers, H.G., 1977. Premotor cortical ablations in monkeys: contralatera changes in visually guided reaching behavior. Science 198, 317-319.

Navas, F., Stark, L., 1968. Sampling or intermittency in hand control system dynamics. Biophys. J. 8, 252-302.

Neggers, S.F., Bekkering, H., 2002. Coordinated control of eye and hand movements in dynamic reaching. Hum. Mov. Sci. 21, 349-376.

Nijhawan, R., 2008. Visual prediction: psychophysics and neurophysiology of compensation for time delays. Behav. Brain Sci. 31, 179-198.

Paillard, J., 1982. The contribution of peripheral and central vision to visually guided reaching. In: Ingle, D.J., Goodale, M.A., Mansfield, R.J.W.(Eds.), Analysis of Visual Behavior. MIT Press, Cambridge, pp. 367-385.

Paré, M., Hanes, D.P., 2003. Controlled movement processing: superior colliculus activity associated with countermanded saccades. J. Neurosci. 23, 6480-6489.

Pastor-Bernier, A., Tremblay, E., Cisek, P., 2012. Dorsal premotor cortex is involved in switching motor plans. Front. Neuroeng. 5, 1-15.

Pelisson, D., Prablanc, C., Goodale, M.A., Jeannerod, M., 1986. Visual control of reaching movements without vision of the limb. II. Evidence of fast unconscious processes correcting the trajectory of the hand to the final position of a doublestep stimulus. Exp. Brain Res. 62, 303-311.

Perenin, M.T., 1997. Optic ataxia and unilateral neglect: clinical evidence for dissociable spatial functions in posterior parietal cortex. In: Thier, H.P., Karnath, H.O. (Eds.), Parietal Lobe Contributions to Orientation in 3D Space. Springer Verlag, Heidelberg, pp. 289-308.

Perenin, M.T., Vighetto, A., 1988. Optic ataxia: a specific disruption in visuomotor mechanisms. Brain 111, 643-674.

Perry, R.J., Zeki, S., 2000. The neurology of saccades and covert shifts in spatia attention: an event-related fMRI study. Brain 123, 2273-2288.

Picton, T.W., Stuss, D.T., Alexander, M.P., Shallice, T., Binns, M.A., Gillingham, S., 2007. Effects of focal frontal lesions on response inhibition. Cereb. Cortex 17, 826-838.

Pisella, L., Gréa, H., Tilikete, C., Vighetto, A., Desmurget, M., Rode, G., Boisson, D. Rosseti, Y., 2000. An 'automatic pilot' for the hand in human posterior parietal cortex: toward reinterpreting optic ataxia. Nat. Neurosci. 3, 729-736.

Pisella, L., Sergio, L., Blangero, A., Torchin, H., Vighetto, A., Rossetti, Y., 2009. Optic ataxia and the function of the dorsal stream: contributions to perception and action. Neuropsychologia 47, 3033-3044.

Port, N.L., Kruse, W., Lee, D., Georgopoulos, A.P., 2001. Motor cortical activity during interception of moving targets. J. Cogn. Neurosci. 13, 306-318.

Prablanc, C., Echallier, J.F., Komilis, E., Jeannerod, M., 1979. Optimal response of eye and hand motor systems in pointing at a visual target: I. Spatio-temporal characteristics of eye and hand movements and their relationships when varying the amount of visual information. Biol. Cybern. 35, 113-124.

Prablanc, C., Martin, O., 1992. Automatic control during hand reaching at undetected two-dimensional target displacements. J. Neurophysiol. 67, 455-469.

Prado, J., Clavagnier, S., Otzenberger, H., Scheiber, C., Kennedy, H., Perenin, M., 2005. Two cortical systems for reaching in central and peripheral vision. Neuron 48 849-858.

Prut, Y., Fetz, E.E., 1999. Primate spinal interneurons show pre-movement instructed delay activity. Nature 401, 590-594.

Quay, H.C., 1997. Inhibition in attention deficit hyperactivity disorder. J. Abnorm. Child Psychol. 25, 7-13.

Ray, S., Pouget, P., Schall, J.D., 2009. Functional distinction between visuomovement and movement neurons in macaque frontal eye field during saccade countermanding. J. Neurophysiol. 102, 3091-3100.

Reichenbach, A., Bresciani, J.P., Peer, A., Bülthoff, H.H., Thielscher, A., 2011. Contributions of the PPC to on-line control of visually guided reaching movements assessed with fMRI-guided TMS. Cereb. Cortex 217, 1602-1612.

Reynolds, C., Ashby, P., 1999. Inhibition in the human motor cortex is reduced just before a voluntary contraction. Neurology 53, 730-735. 
Rossetti, Y., Pisella, L., Vighetto, A., 2003. Optic ataxia revisited: visually guided action versus immediate visuomotor control. Exp. Brain Res. 153, 171-179.

Rubia, K., Russell, T., Overmeyer, S., Brammer, M.J., Bullmore, E.T., Sharma, T., Simmons, A., Williams, S.C., Giampietro, V., Andrew, C.M., Taylor, E., 2001. Mapping motor inhibition: conjunctive brain activations across different versions of go/no-go and stop tasks. Neuroimage 13, 250-261.

Sabes, P.N., 2000. The planning and control of reaching movements. Curr. Opin. Neurobiol. 10, 740-746.

Sabes, P.N., 2011. Sensory integration for reaching: models of optimality in the context of behavior and the underlying neural circuits. Prog. Brain Res. 191, 195-209.

Safavynia, S.A., Ting, L.H., 2013. Sensorimotor feedback based on task-relevant error robustly predicts temporal recruitment and multidirectional tuning of muscle synergies. J. Neurophysiol. 109, 31-45.

Sailer, U., Eggert, T., Ditterich, J., Straube, A., 2000. Spatial and temporal aspects of eye-hand coordination across different tasks. Exp. Brain Res. 134, 163-173.

Salenius, S., Portin, K., Kajola, M., Salmelin, R., Hari, R., 1997. Cortical control of human motoneuron firing during isometric contraction. J. Neurophysiol. 77, 3401-3405.

Sallet, J., Mars, R.B., Noonan, M.A.P., Neubert, F-X., Jbabdi, S., O’Reilly, J.X., Filippini, N. Thomas, A.G., Rushworth, M.F. 2013. The organization of dorsal frontal cortex in humans and macaques. J. Neurosci. 33, 12255-12274.

Saunders, J.A., Knill, D.C., 2003. Humans use continuous visual feedback from the hand to control fast reaching movements. Exp. Brain Res. 152, 341-352.

Sasaki, K., Gemba, H., 1986. Electrical activity in the prefrontal cortex specific to nogo reaction of conditioned hand movement with colour discrimination in the monkey. Exp. Brain Res. 164, 603-606.

Sasaki, K., Gemba, H., Tsujimoto, T., 1989. Suppression of visually initiated hand movement by stimulation of the prefrontal cortex in the monkey. Brain Res. 495, 100-107.

Sawaguchi, T., Yamane, I., Kubota, K., 1996. Application of the GABA antagonist bicuculline to the premotor cortex reduces the ability to withhold reaching movements by well-trained monkeys in visually guided reaching task. J. Neurophysiol. 75, 2150-2156.

Scangos, K.W., Aronberg, R., Stuphorn, V., 2013. Performance monitoring by presupplementary and supplementary motor area during an arm movement countermanding task. J. Neurophysiol. 109, 1928-1939.

Scangos, K.W., Stuphorn, V., 2010. Medial frontal cortex motivates but does not control movement initiation in the countermanding task. J. Neurosci. 30, 1968-1982.

Schall, J.D., 2001. Neural basis of deciding, choosing and acting. Nat. Rev. Neurosci. $2,33-42$.

Schall, J.D., Godlove, D.C., 2012. Current advances and pressing problems in studies of stopping. Curr. Opin. Neurobiol. 22, 1-10.

Schiller, P.H., Malpeli, J.G., 1977. Properties and tectal projections of monkey retinal ganglion cells. J. Neurophysiol. 40, 428-445

Schiller, P.H., Malpeli, J.G., Schein, S.J., 1979. Composition of geniculostriate input to superior colliculus of the rhesus monkey. J. Neurophysiol. 42, 1124-1133.

Schlag, J., Schlag-Rey, M., 2002. Through the eye, slowly: delays and localization errors in the visual system. Nat. Rev. Neurosci. 3, 191-200.

Schmidt, A., Walter, M., Gerber, H., Schmid, O., Smieskova, R., Bendfeldt, K., Wiesbeck, G.A., Riecher-Rössler, A, Lang, U.E, Rubia, K., McGuire, P., Borgwardt, S., 2013. Inferior frontal cortex modulation with an acute dose of heroin during cognitive control. Neuropsychopharmacology 38, 2231-2239.

Shadmehr, R., Mussa-Ivaldi, S., 2012. Biological Learning and Control: How the Brain Builds Representations, Predicts Events, and Makes Decisions. MIT Press, Cambridge, MA.

Shadmehr, R., Wise, S.P., 2005. Computational Neurobiology of Reaching and Pointing: A Foundation for Motor Learning. MIT Press, Cambridge, MA.

Shallice, T., 1988. From Neuropsychology to Mental Structure. Cambridge University Press, UK.

Shallice, T., Cooper, R.P., 2011. The Organisation of Mind. Oxford University Press, UK.

Shallice, T., Mussoni, A., D’Agostino, S., Skrap, M., 2010. Right posterior cortical functions in a tumour patient series. Cortex 46, 1178-1188.

Sharp, D.J., Bonnelle, V., De Boissezon, X., Beckmann, C.F., James, S.G., Patel, M.C., Mehta, M.A., 2010. Distinct frontal systems for response inhibition, attentional capture, and error processing. Proc. Natl. Acad. Sci. U.S.A. 107, 6106-6111.
Shenoy, K.V., Sahani, M., Churchland, M.M., 2013. Cortical control of arm movements: a dynamical systems perspective. Annu. Rev. Neurosci. 36, 337-359.

Soechting, J.F., Flanders, M., 1992. Moving in three-dimensional space: frames of reference, vectors, and coordinate systems. Annu. Rev. Neurosci. 15, 167-191.

Soechting, J.F., Lacquaniti, F., 1983. Modification of trajectory of a pointing movement in response to a change in target location. J. Neurophysiol. 49, 548-564.

Striemer, C.L., Yukovsky, J., Goodale, M.A., 2010. Can intention override the "automatic pilot"? Exp. Brain Res. 202, 623-632

Stuphorn, V., Emeric, E.E., 2012. Proactive and reactive control by the medial frontal cortex. Front. Neuroeng. 5, 9.

Sundberg, K.A., Fallah, M., Reynolds, J.H., 2006. A motion-dependent distortion of retinotopy in area V4. Neuron 49, 447-457.

Swick, D., Ashley, V., Turken, U., 2011. Are the neural correlates of stopping and not going identical? Quantitative meta-analysis of two response inhibition tasks. Neuroimage 56, 1655-1665.

Tanji, J., Kurata, K., 1985. Contrasting neuronal activity in supplementary and precentral motor cortex of monkeys. I. Responses to instructions determining motor responses to forthcoming signals of different modalities. J. Neurophysiol. 53, 129-141.

Todorov, E., Jordan, M.I., 2002. Optimal feedback control as a theory of motor coordination. Nat. Neurosci. 5, 1226-1235.

Tokuno, H., Nambu, A., 2000. Organization of nonprimary motor cortical inputs on pyramidal and nonpyramidal tract neurons of primary motor cortex: an electrophysiological study in the macaque monkey. Cereb. Cortex 10, 58-68.

Tomasi, S., Caminiti, R., Innocenti, G.M., 2012. Areal differences in diameter and length of corticofugal projections. Cereb. Cortex 22, 1463-1472.

Trillenberg, P., Sprenger Petersen, D., Komp, D., Heide, W., Helmchen, C., 2007. Functional dissociation of saccade and hand reaching control with bilateral lesions of the medial wall of the intraparietal sulcus: implications for optic ataxia. Neuroimage 36, T69-T76.

van de Kamp, C., Gawthrop, P.J., Gollee, H., Loram, I.D., 2013. Refractoriness in sustained visuo-manual control: is the refractory duration intrinsic or does it depend on external system properties? PLoS Comput. Biol. 9, e1002843.

van Donkelaar, P., Lee, J.H., Drew, A.S., 2000. Transcranial magnetic stimulation disrupts eye-hand interactions in the posterior parietal cortex. J. Neurophysiol. 84, 1677-1680.

van Sonderen, J.F., Gielen, C.C., Denier van der Gon, J.J., 1989. Motor programmes for goal-directed movements are continuously adjusted according to changes in target location. Exp. Brain Res. 78, 139-146.

Verbruggen, F., Aron, A.R., Stevens, M.A., Chambers, C.D., 2010. Theta burst stimulation dissociates attention and action updating in human inferior frontal cortex. Proc. Natl. Acad. Sci. U.S.A. 107, 13966-13971.

Verbruggen, F., Logan, G.D., 2009. Models of response inhibition in the stop signal and stop change paradigms. Neurosci. Biobehav. Rev. 33, 647-661.

Verfaellie, M., Heilman, K.M., 1987. Response preparation and response inhibition after lesions of the medial frontal lobe. Arch. Neurol. 44, 1265-1271.

Vigneswaran, G., Kraskov, A., Lemon, R.N., 2011. Large identified pyramidal cells in macaque motor and premotor cortex exhibit thin spikes: implications for cel type classification. J. Neurosci. 31, 14235-14242.

Vince, M.A., 1948. The intermittency of control movements and the psychological refractory period. Br. J. Psychol. Gen. Sect. 38, 149-157.

Vink, M., Kahn, R.S., Raemaekers, M., van den Heuvel, M., Boersma, M., Ramsey, N.F. 2005. Function of striatum beyond inhibition and execution of motor responses. Hum. Brain Mapp. 25, 336-344.

Watanabe, M., 1986. Prefrontal unit activity during delayed conditional go/no-go discrimination in the monkey. II. Relation to go and no-go responses. Brain Res. $382,15-27$.

Welford, A.T., 1952. The psychological refractory period and the timing of high speed performance. A review and theory. Br. J. Psychol. 43, 2-19.

Wise, S.P., 1985. The primate premotor cortex: past, present and preparatory. Annu. Rev. Neurosci. 8, 1-19.

Wolpert, D.M., Goodbody, S.J., Husain, M., 1998. Maintaining internal representations: the role of the human superior parietal lobe Nat Neurosci. 6, 529-533.

Wolpert, D.M., Miall, R.C., 1996. Forward models for physiological motor control Natl. Netw. 9, 1265-1279.

Zhang, Y., Chen, Y., Bressler, S.L., Ding, M., 2008. Response preparation and inhibition: the role of the cortical sensorimotor beta rhythm. Neuroscience 156 238-246. 